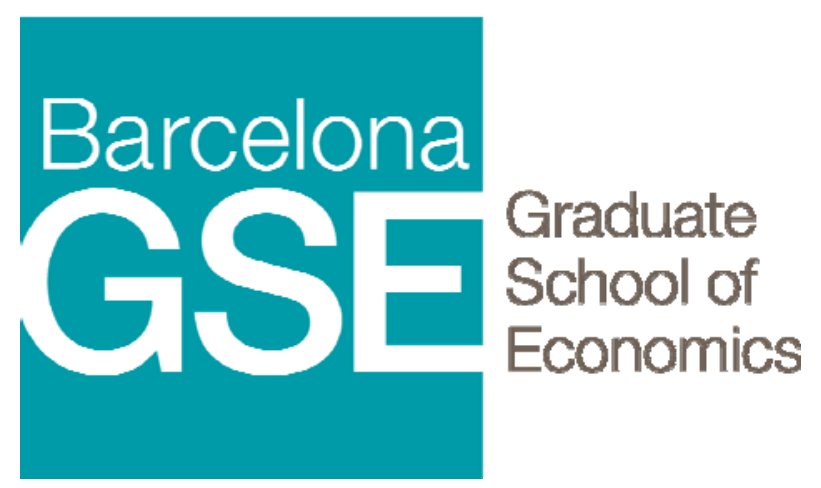

\title{
Immunity to Credible Deviations from the Truth
}

\author{
Salvador Barberà \\ Dolors Berga \\ Bernardo Moreno
}

This version: January 2017

(April 2016)

Barcelona GSE Working Paper Series

Working Paper no 893 


\title{
Immunity to credible deviations from the truth
}

\author{
Salvador Barberà \\ MOVE, Universitat Autònoma de Barcelona, and Barcelona GSE. Departament d'Economia i \\ d'Història Econòmica, Edifici B, 08193 Bellaterra, Spain. E-mail: salvador.barbera@uab.cat \\ Dolors Berga* \\ Departament d'Economia, C/ Universitat de Girona, 10; Universitat de Girona, 17003 Girona, \\ Spain. E-mail: dolors.berga@udg.edu \\ Bernardo Moreno \\ Departamento de Teoría e Historia Económica, Facultad de Ciencias Económicas y Empresariales, \\ Universidad de Málaga, Campus de El Ejido, 29071 Málaga, Spain. E-mail: bernardo@uma.es
}

January, 6th 2017

\begin{abstract}
We study a notion of non-manipulability by groups, based on the idea that only some agreements among potential manipulators may be credible. The derived notion of immunity to credible manipulations by groups is intermediate between individual and group strategy-proofness. Our main non-recursive definition turns out to be equivalent, in our context, to the requirement that truthful preference revelation should be a strong coalitionproof equilibrium, as recursively defined by Peleg and Sudhölter $(1998,1999)$. We provide characterizations of strategy-proof rules separating those that satisfy it from those that do not for a large family of public good decision problems.

Journal of Economic Literature Classification Numbers: D71.

Keywords: Strategy-proofness, Group strategy-proofness, Credibility, Voting.
\end{abstract}

\footnotetext{
*Corresponding author: E-mail: dolors.berga@udg.edu
} 


\section{Introduction}

In many contexts where the basic incentive property of strategy-proofness can be met by non-trivial social choice functions, it becomes natural to investigate whether some of them may not only be immune to manipulation by individuals, but can also resist manipulation by groups of coordinated agents. In previous work (Barberà, Berga, and Moreno, 2010 and 2016) we have identified conditions under which, surprisingly, all social choice functions that are immune to manipulations by individuals will also be free from group manipulation. But this is not always the case. In particular, many interesting strategy-proof rules in separable environments ${ }^{1}$ will indeed be group manipulable. In these cases, we shall argue that not all group manipulations represent an equally serious threat, because some strategic movements by coalitions are credible, while others are not. To make this point precise, we define several notions of immunity to credible group manipulations and characterize subclasses of social choice rules that satisfy them in specific environments. We concentrate especially in the following: we say that a deviation leading to a profitable improvement for a group is credible if no individual member of the group would gain from not following the agreed upon strategy under the assumption that all others stick to the agreement. Hence, a group manipulation is credible if the set of prescribed strategies for those individuals who plan to deviate are a Nash equilibrium in the induced game where these agents could use any other preference instead, while those of the rest of agents remain fixed. ${ }^{2}$ And then we say that a rule is immune to credible group manipulations if no set of agents can find a profitable deviation away from the truth that is credible. We illustrate the strength of our new definition, which is more demanding than individual but weaker than group strategy-proofness, by characterizing some families of rules in separable environments, and distinguishing between those that can meet our new requirement and those that cannot. The issue of credibility of group deviations has been formalized in alternative ways, one of which is based on the recursive definition of coalition-proof Nash equilibrium (see Bernheim et al., 1987). In fact, Peleg and Sudhölter (1999) applied this concept to the same environment that we analyze, and concluded that all rules that are strategyproof in that environment are also coalition-proof. In the same paper, these authors (see also Peleg, 1998) define strong coalition proofness, again recursively based. Surprisingly, this turns out to be equivalent, in our context, to our non-recursive concept of immunity. Let us remark again that our notion of immunity, and that of strong coalitionproofness, allows for a classification and characterization of different strategy-proof rules according to their degree of group manipulability.

After this Introduction we provide notation and definitions in Section 2. Section 3 presents characterization results in two specific contexts. We start with the problem faced by voters who must select a set of entrants to a club, as described in Barberà, Sonnenschein, and Zhou (1991). We concentrate on quota rules: voters can support all candidates they like, and then those who receive at least a fixed number of votes, $q$, are chosen. In the domain of

\footnotetext{
${ }^{1}$ We use this expression loosely here. Formal definitions of the environments we refer to are given in the Section 3.

${ }^{2}$ Actually, the concept remains the same if the possible deviations of manipulators are limited to either following the prescription or revealing their true preferences. See Section 4 for a deeper discussion of this and related points.
} 
separable preferences, we show that rules based in quota 1 or $n$ (where $n$ is the number of voters) are immune to credible deviations, while all other rules in the class are not. Hence, very extreme distributions of power among voters are needed to guarantee immunity. We then turn to a general version of choice among multi-dimensional alternatives under separable preferences, also called multidimensional single-peaked. We build on Moulin (1980), Border and Jordan (1983) and Barberà, Gul, and Stacchetti (1993). The cases we consider include the previous example and many more. We restrict attention to a large class of rules that are strategy-proof in this context, and again characterize those within the class that are immune to credible deviations by groups. Again, a requirement in the form of unanimity plays a crucial role in separating these rules for all the rest, those that are credibly manipulable. Section 4 discusses alternative definitions of credibility for group manipulations, establishes the equivalence of several apparently different formulations, and the differences with other potential definitions, whose proofs are also examined. Section 5 concludes with some final remarks. The Appendix contains proofs that are not essential for the continuity of our arguments.

\section{The model and definitions: immunity and credibility}

Let $N=\{1, \ldots, n\}$ be the set of agents and $A$ be the set of alternatives. Preferences are complete, reflexive, and transitive binary relations on alternatives. Let $\mathcal{U}$ denote such set of preferences. For $i \in N, R_{i}$ denotes agent $i$ 's preferences on $A$. As usual, $P_{i}$ and $I_{i}$ denotes the strict and indifference preference relation induced by $R_{i}$, respectively. A preference profile $R_{N}=\left(R_{1}, \ldots, R_{n}\right) \in \mathcal{U} \times \ldots \times \mathcal{U}=\mathcal{U}^{n}$ is a $n$-tuple of preferences on $A$. It can also be represented by $R_{N}=\left(R_{C}, R_{N \backslash C}\right) \in \mathcal{U}^{n}$ when we want to stress the role of coalition $C$ in $N$. We call a subprofile of agents in $C$ as $R_{C} \in \times_{i \in C} \mathcal{U}=\mathcal{U}^{c}$.

A social choice function (or rule) $f$ on $\mathcal{U}^{n}$ is a function $f: \mathcal{U}^{n} \rightarrow A$.

At this point it is worth mentioning that although we define our main concept and state our results in Sections 2 and 4 assuming that the set of preferences is the same for all agents, all definitions and results would be correct and straightforwardly obtained if we allowed agents' sets of preferences to be different. We assume equal sets of preferences since this is the case of our application in Section 3.

Let us define some incentive-related properties of social choice functions. The best known non-manipulability axiom is that of strategy-proofness. In its usual form it requires the truth to be a dominant strategy for each agent. However, we provide a more general definition which encompasses strategy-proofness and also considers the option that several agents evaluate the possibility of joint deviations.

Definition 1 Let $f$ be a social choice function on $\mathcal{U}^{n}$. Let $R_{N} \in \mathcal{U}^{n}$ and $C \subseteq N$. A subprofile $R_{C}^{\prime} \in \mathcal{U}^{c}$ such that $R_{i}^{\prime} \neq R_{i}$ for all $i \in C$ is a profitable deviation of coalition $C$ against profile $R_{N}$ (for $f$ ) if $f\left(R_{C}^{\prime}, R_{N \backslash C}\right) P_{i} f\left(R_{N}\right)$ for any agent $i \in C$.

Profitable deviations are usually called (group) manipulations in the standard definitions of group and individual strategy-proofness. Throughout the paper we shall assume that 
among profitable deviations for single agents there is always one that is best. ${ }^{3}$

Definition $2 A$ social choice function $f$ on $\mathcal{U}^{n}$ is manipulable at $R_{N} \in \mathcal{U}^{n}$ by coalition $C \subseteq N$ if there exists a profitable deviation of coalition $C$ against profile $R_{N}$, say $R_{C}^{\prime} \in \mathcal{U}^{c}$. A social choice function is group strategy-proof if it is not manipulable by any coalition $C \subseteq N$.

When we consider only deviations by single agent coalitions we have strategy-proofness.

Definition 3 A social choice function $f$ on $\mathcal{U}^{n}$ is manipulable at $R_{N} \in \mathcal{U}^{n}$ by agent $i \in N$ if there exists a profitable deviation of agent $i$ against profile $R_{N}$, say $R_{i}^{\prime} \in \mathcal{U}$. A social choice function is strategy-proof if it is not manipulable by any agent $i \in N$.

Remark that, formally, strategy-proofness is a much weaker condition than group strategyproofness in any of its versions. In many environments and in spite of this definitional gap, individual strategy-proof rules end up also being group strategy-proof. ${ }^{4}$ But, of course, in many other situations this equivalence may not hold, and even when there are attractive strategy-proof rules, they are open to manipulation by groups. In this paper, we concentrate on a form of manipulation that is intermediate between those of individual and group strategy-proofness and that is based on the notion of credible profitable deviations, those where no agent in the deviating coalition can gain by not declaring those preferences she was supposed to use as part of the group strategy. Formally,

Definition 4 Let $f$ be a social choice function on $\mathcal{U}^{n}$. Let $R_{N} \in \mathcal{U}^{n}$ and $C \subseteq N$. We say that $R_{C}^{\prime} \in \mathcal{U}^{c}$ a profitable deviation of $C$ against $R_{N}$ is credible if for all $i \in C$ and all $\bar{R}_{i} \in \mathcal{U}$, then $f\left(R_{C}^{\prime}, R_{N \backslash C}\right) R_{i} f\left(\bar{R}_{i}, R_{C \backslash\{i\}}^{\prime}, R_{N \backslash C}\right)$.

On other terms, a profitable deviation by $C$ from $R_{N}=\left(R_{C}, R_{N \backslash C}\right)$ is credible if $R_{C}^{\prime}$ is a Nash equilibrium of the game among agents in $C$, when these agents strategies are their admissible preferences and the outcome function is $f\left(\cdot, R_{N \backslash C}\right)$.

Definition 5 A social choice function $f$ on $\mathcal{U}^{n}$ is immune to credible deviations if for any $R_{N} \in \mathcal{U}^{n}$, any $C \subseteq N$, there is no credible profitable deviation of $C$ against $R_{N}$ (that is, for any profitable deviation $R_{C}^{\prime} \in \mathcal{U}^{c}$ of $C$ against $R_{N}$ there exists $i \in C$ such that $f\left(\bar{R}_{i}, R_{C \backslash\{i\}}^{\prime}, R_{N \backslash C}\right) P_{i} f\left(R_{C}^{\prime}, R_{N \mid C}\right)$ for some $\left.\bar{R}_{i} \in \mathcal{U}\right) .^{5}$

Immunity to credible deviations means that no profitable deviation of any coalition is credible at any profile. Observe that group strategy-proofness implies immunity to credible deviations as defined above. However, in general the converse implication fails (see Proposition 1 below). Moreover, as Lemma 1 shows, immunity to credible deviations implies strategy-proofness. And strategy-proofness implies immunity to credible deviations by singletons.

\footnotetext{
${ }^{3}$ The existence of a best deviation is guaranteed when the number of alternatives, and those of preferences are finite. Moreover, the condition will also hold under standard assumptions.

${ }^{4}$ See Le Breton and Zaporozhets (2009), Barberà, Berga, and Moreno (2010), and Barberà, Berga, and Moreno (2016).

${ }^{5}$ For short, we use the expression "immunity to credible deviations" instead of "immunity to credible profitable deviations" since, by Definition 4, a credible deviation is profitable.
} 
Lemma 1 Any social choice function $f$ on $\mathcal{U}^{n}$ that is immune to credible deviations is strategy-proof.

Proof. By contradiction, let $R_{N} \in \mathcal{U}^{n}, i \in N$, and $R_{i}^{\prime} \in \mathcal{U}$ such that $R_{i}^{\prime} \neq R_{i}$ and $f\left(R_{i}^{\prime}, R_{N \backslash\{i\}}\right) P_{i} f\left(R_{N}\right)$ and $R_{i}^{\prime}$ be such that it is a best deviation for agent $i$ (which, as already stated, we assume to exist). By immunity to credible deviations, there exists $\bar{R}_{i} \in \mathcal{U}$, such that $f\left(\bar{R}_{i}, R_{N \backslash\{i\}}\right) P_{i} f\left(R_{i}^{\prime}, R_{N \backslash\{i\}}\right)$ which contradicts that $R_{i}^{\prime}$ is a best deviation for $i$.

\section{Applications}

We have remarked in the introduction that in some important domains one can define strategy-proof rules that are, however, not group strategy-proof. In this section we illustrate the strength of our new definition, by showing that it allows to differentiate between different rules that are all manipulable by groups, but with different degrees of credibility. In fact, we can characterize the subfamilies of strategy-proof, anonymous, and onto rules that are immune to credible group manipulations, and separate them from those that are not.

We concentrate on the analysis of strategy-proof rules in contexts where alternatives are multidimensional and preferences are multidimensional single-peaked, since it is a very well studied case admitting a large family of non-trivial, strategy-proof social choice rules which are, nonetheless, group manipulable. Hence, it is a natural testing ground for our presumption that some of them may be immune to credible manipulations while others are not.

For expositional purposes, we have chosen to first discuss a special case of the general model, one where alternatives are sets of candidates, preferences are over sets and satisfy a separability condition. After that, we turn to the general case. The reader who prefers to stick to the initial example can already appreciate the broad lines of the arguments leading to our characterizations. Likewise, the reader who prefers the general (and somewhat more involved) arguments may want to skip the special case.

Now, let us comment that the distinction between those strategy-proof rules in our context that are immune to group deviations and those that do not depend on the existence of some "privileged" alternative in each dimension that will be chosen unless all agents agree otherwise. ${ }^{6}$ In that limited sense, our immune rules satisfy a form of solidarity among agents that has been presented as a normative requirement in a different context (Thomson, 1993, 1999). However, we prefer to remain agnostic regarding the desirability of using those rules, rather than others that may be vulnerable to credible group deviations but provide a more even treatment to different alternatives. At any rate, our characterization is based on remarks that hinge upon our own definition of credibility and do not depend on any normative considerations.

Let us anticipate the reasons why we may be able to avoid credible deviations under certain rules and not in others. The explanation at this point will necessarily be sketchy,

\footnotetext{
${ }^{6}$ To be more precise, observe that for the setting studied in Section 3.2, such privileged alternative may not exist in one dimension. This is because in the one dimensional setting each strategy-proof rule is also group strategy-proof.
} 
as it comes before formal statements, but we hope it may help the reader. Take an agent $i$ who is considering to participate in a profitable deviation involving agents in coalition $S$, and in case of participation is asked to reveal a preference $R_{i}^{\prime}$ rather than her true preference $R_{i}$. Suppose that, if all other members of the deviating coalition do follow the strategies they are asked to, then $i$ will not be able to alter the resulting outcome, regardless of whether she still declares $R_{i}^{\prime}$ or any other preference. Then we can say that this agent is individually redundant. And if all agents involved in a jointly beneficial deviation are individually redundant in that sense, their joint beneficial actions will constitute a Nash equilibrium and their threat will be credible. Now, how can one make voters redundant? By creating coalitions and strategies that support their objectives "in excess". Say that they need one agent to deviate in a certain manner, but actually ask two of them to do it. Then, each one becomes "redundant" for the purposes of the decision, but not of course for the credibility of the threat. Our reasoning along the proofs that follow goes in that direction. Rules that allow for threats to be reinforced by involving more agents than those who are strictly necessary to obtain a gain will be vulnerable to credible deviations.

After these motivational comments, we introduce our general framework and definitions.

Let $\mathcal{K}=\{1, \ldots, K\}$ be a finite set of $K \geq 2$ coordinates and for each $k \in \mathcal{K}$, let $B_{k}=$ $\left[a_{k}, b_{k}\right]$ with $a_{k}<b_{k}$ be an integer interval. Our alternatives are $K$-dimensional vectors in $B=\prod_{k=1}^{K} B_{k}$. To stress the role of a set of coordinates $k_{S}$, we will write $x=\left(x_{k_{S}}, x_{\mathcal{K} \backslash k_{S}}\right) \in B$. We endow $B$ with the $L_{1}$-norm. That is, for any $x \in B$,

$$
\|x\|=\sum_{k=1}^{K}\left|x_{k}\right| .
$$

Given $x, y \in B$, the minimal box containing $x$ and $y$ is defined by

$$
M B(x, y)=\{z \in B:\|x-y\|=\|x-z\|+\|z-y\|\} .
$$

We restrict attention to the case where individual preferences are antisymmetric and thus, have a unique best alternative that we denote by $\tau\left(R_{i}\right)$.

We now impose a restriction on preferences which is a natural extension of singlepeakedness to the multidimensional setting.

Definition 6 A preference $R_{i} \in \mathcal{U}$ is multidimensional single-peaked if for any $z, y \in B$, if $y \in M B\left(z, \tau\left(R_{i}\right)\right)$ then $y R_{i} z$.

Let $\mathcal{S} \subset \mathcal{U}$ be the set of multidimensional single-peaked preferences on $B$. Under this preference restriction $\tau\left(R_{i}\right)=\left(\tau_{1}\left(R_{i}\right), \ldots, \tau_{K}\left(R_{i}\right)\right) \in B$ where $\tau_{j}\left(R_{i}\right)$ is the best (or top) alternative of $R_{i}$ in dimension $j$.

Multidimensional single-peakedness has two basic implications. One is that the restriction of preferences to alternatives that only differ in a single dimension, while holding the values in all other dimensions fixed, has its best element at the same value than the absolute best alternative. Informally, the projection of the top in any dimension is the top of the projection; and this is independent of the values at which we may have fixed the rest of dimensions. The other implication is that any of these one-dimensional restrictions is single-peaked. 
It is known in the literature (Barberà, Gul, and Stacchetti, 1993) that the class of multidimensional Generalized Median Voter Schemes (GMVS) are the only strategy-proof social choice functions in our setting, where multidimensional GMVS can be written as $K$ unidimensional GMVS, one for each dimension. In this paper we restrict attention to a particular subclass of GMVS that is a $K$-dimensional extension of what Moulin (1980) called generalized Condorcet winner rules.

For each $k \in \mathcal{K}$, let $P_{k}=\left\{p_{k}^{1}, \ldots, p_{k}^{n-1}\right\}$ be an ordered list of $n-1$ values in $B_{k}$ where $p_{k}^{1} \leq \ldots \leq p_{k}^{n-1}$. In what follows, we shall use $K$ lists of such values, one for each dimension, as definitional parameters.

Definition 7 We say that $f: \mathcal{S}^{n} \rightarrow B, f=\left(f_{1}, \ldots, f_{K}\right)$ is a generalized Condorcet winner rule if for any profile $R_{N} \in \mathcal{S}^{n}$, for any $k \in \mathcal{K}, f_{k}\left(R_{N}\right)=\operatorname{med}\left\{\tau_{k}\left(R_{1}\right), \ldots, \tau_{k}\left(R_{n}\right), p_{k}^{1}, \ldots, p_{k}^{n-1}\right\}$, where $P_{k}(f)=\left\{p_{k}^{1}, \ldots, p_{k}^{n-1}\right\}$ is a list of parameters in $B_{k}{ }^{7}$

Remark 1. Generalized Condorcet winner rules constitute the set of anonymous, onto, strategy-proof rules in multidimensional single-peaked domains (see Barberà, Gul, and Stacchetti, 1993).

Remark 2. When all parameters in Definition 7 have the same value for some dimension $k$, we say for short that the list of parameters is degenerate in that dimension. That does not mean, however, that these rules are not interesting. See our discussion in Section 5 .

\subsection{Choosing sets of candidates}

Before engaging in a full analysis of those rules that are immune in a general framework, we consider a simple case proposed in Barberà, Sonnenschein, and Zhou (1991). These authors discuss situations where there exists a set $\mathcal{O}$ of $K$ potential candidates or objects out of which a set of agents must choose the new members of a club, and they characterize the voting rules that may be strategy-proof when preferences are separable. For the benefit of the reader who prefers to stick to this simple case, we analyze it separately, but let us start by saying that it is simply a special case of our more general result discussed in the following subsection. This is because any set of candidates can be described by its characteristic function, assigning value 1 to those that are in the set and 0 to those outside it. Hence, in terms of the alternatives, this is a special case where $B_{k}$ can take only two values, 0 and 1 , in each dimension. As for the restriction of preferences, their notion of separability is equivalent to multidimensional single-peakedness when adapted to their limited context. ${ }^{8}$ As we have observed for the general case, here again the best element in any dimension will be found at the value of the absolute best in that dimension (now 0 or 1 ), and the implication of single-peakedness is immediate because the variable in each dimension only takes two values.

Individual preferences are linear orders on the set $2^{\mathcal{O}}$ (including the empty set). Given any preference $R$ on $2^{\mathcal{O}}$, we define the set of "good" objects $G(\mathcal{O}, R)=\left\{o_{k} \in \mathcal{O}:\left\{o_{k}\right\} P \varnothing\right\}$ and the set of "bad" objects $\mathcal{O} \backslash G(\mathcal{O}, R)=\left\{o_{k} \in \mathcal{O}: \varnothing P\left\{o_{k}\right\}\right\}$.

\footnotetext{
${ }^{7}$ The notation med denotes the median(s) of an ordered list. In the present definition this will be unique.

${ }^{8}$ See also Border and Jordan (1983), Le Breton and Sen (1999), Le Breton and Weymark (1999) who have analyzed a model with separable preferences in continuous multidimensional spaces.
} 
Definition $8 R$ is a separable preference on $2^{\mathcal{O}}$ if and only if for any set $T$ and any object $o_{l} \notin T, T \cup\left\{o_{l}\right\} P T$ if $o_{l} \in G(\mathcal{O}, R)$.

In words, adding a new good object to any set makes the union better than the original set and adding a bad object makes it worse. Now $\mathcal{S}$ denote the set of all separable preferences.

In this setting there exist strategy-proof social choice functions. In particular, the set of such functions that are anonymous, neutral, and satisfy voter sovereignly coincides with the family of voting by quota rules, $f: \mathcal{S}^{n} \rightarrow 2^{\mathcal{O}}$ defined as follows:

Definition 9 Let $q \in\{1, \ldots, n\}$. The social choice function $f$ on $\mathcal{S}^{n}$ defined so that for any $R_{N} \in \mathcal{S}^{n}$

$$
f\left(R_{N}\right)=\left\{o_{k} \in \mathcal{O}:\left|\left\{i: o_{k} \in G\left(\mathcal{O}, R_{i}\right)\right\}\right| \geq q\right\}
$$

is called voting by quota $q$.

However, none of these voting by quota rules are group strategy-proof. And yet, we will show that some of them are immune to credible deviations, while others are not.

Before providing a characterization theorem allowing to distinguish between those rules that are immune and those that are not, we present two examples with 5 voters and 2 candidates. The set of all separable preferences when $K=2$ is detailed in Table 1 .

\begin{tabular}{|c|c|c|c|c|c|c|c|}
\hline$R^{1}$ & $R^{2}$ & $R^{3}$ & $R^{4}$ & $R^{5}$ & $R^{6}$ & $R^{7}$ & $R^{8}$ \\
\hline$\varnothing$ & $\varnothing$ & $o_{1}$ & $o_{1}$ & $o_{2}$ & $o_{2}$ & $\left\{o_{1}, o_{2}\right\}$ & $\left\{o_{1}, o_{2}\right\}$ \\
\hline$o_{1}$ & $o_{2}$ & $\varnothing$ & $\left\{o_{1}, o_{2}\right\}$ & $\varnothing$ & $\left\{o_{1}, o_{2}\right\}$ & $o_{1}$ & $o_{2}$ \\
\hline$o_{2}$ & $o_{1}$ & $\left\{o_{1}, o_{2}\right\}$ & $\varnothing$ & $\left\{o_{1}, o_{2}\right\}$ & $\varnothing$ & $O_{2}$ & $O_{1}$ \\
\hline$\left\{o_{1}, o_{2}\right\}$ & $\left\{o_{1}, o_{2}\right\}$ & $o_{2}$ & $o_{2}$ & $o_{1}$ & $o_{1}$ & $\varnothing$ & $\varnothing$ \\
\hline
\end{tabular}

Table 1. The set of all separable preferences when $K=2$.

Example 1 Voting by quota 1: each agent declares her best set of objects and any object that is declared as good by some agent is selected.

Consider the profile where $R_{1}=R^{3}, R_{2}=R^{5}$ and for any other agent $R_{i}=R^{1}$ the outcome would be $\left\{o_{1}, o_{2}\right\}$, whereas 1 and 2 could vote for $\varnothing$ and get a preferred outcome.

This proves that the rule is group manipulable. Notice, however, that after having agreed on voting for empty, any of the two agents could simply keep voting for their preferred candidate, and obtain an even better result, provided the other sticks to her announcement. Hence, this group manipulation will not be credible. We leave it to the reader to check that any other group manipulation under this rule will fail to be credible. Hence, in this example, voting by quota 1 is immune to credible deviations. As we shall see the result generalizes.

Example 2 Voting by quota 3: each agent declares her best set of objects and any object that is declared as good by at least three agents is selected.

Consider now the profile where $R_{1}=R_{2}=R^{3}, R_{3}=R_{4}=R^{5}$ and $R_{5}=R^{7}$. Then, the outcome would be $\left\{o_{1}, o_{2}\right\}$. Now, if agents 1 and 2 agree to vote for $\varnothing$, and so do agents 3 and 4 , the coalition of these four agents can manipulate and have the outcome to 
be $\varnothing$, that they all prefer to $\left\{o_{1}, o_{2}\right\}$. Hence, the rule is group manipulable. Moreover, this particular manipulation is credible, because as long as the rest of deviators complies with the agreement, no single agent can profitable deviate from it. Hence, the rule is not immune to credible deviations in this case.

Notice, however, that there would be other profitable deviations that would not be credible. For example, the one where only 1 and 3 agreed to drop their support to their preferred alternative.

In fact, we can prove the following general result.

Proposition 1 Let $n=2$ or $n>3$. Then, voting by quota 1 and $n$ are the only voting by quota rules satisfying immunity to credible deviations.

Proof. To prove that voting by quota 1 is never subject to credible profitable deviations, notice that any profitable deviation by a group must involve agents who do not vote for some of the candidates they like (since they can always get them without anyone's help). In exchange these agents can get others not to vote for candidates that they dislike.

Let $R_{N} \in \mathcal{S}^{n}, C$ be a coalition that has a profitable deviation $R_{C}^{\prime}$ against $R_{N}$. Note that $f\left(R_{N}\right) \varsubsetneqq f\left(R_{C}^{\prime}, R_{N \backslash C}\right)$ (otherwise, if $f\left(R_{N}\right) \varsubsetneqq f\left(R_{C}^{\prime}, R_{N \backslash C}\right)$, by quota 1 , for any candidate $o \in f\left(R_{C}^{\prime}, R_{N \backslash C}\right) \backslash f\left(R_{N}\right), o \notin G\left(\mathcal{O}, R_{i}\right)$ for any $i \in N$. By separability, for any $i \in N$, $f\left(R_{N}\right) P_{i} f\left(R_{C}^{\prime}, R_{N \backslash C}\right)$ and $R_{C}^{\prime}$ could not be a profitable deviation, which is a contradiction). Thus, there exists a candidate $o$ such that $o \in f\left(R_{N}\right) \backslash f\left(R_{C}^{\prime}, R_{N \backslash C}\right)$. Observe that for each such candidate $o \in f\left(R_{N}\right) \backslash f\left(R_{C}^{\prime}, R_{N \backslash C}\right)$, since $f$ is voting by quota 1 , there is at least one individual $i \in C$ such that $o \in G\left(\mathcal{O}, R_{i}\right)$ and $o \notin G\left(\mathcal{O}, R_{i}^{\prime}\right)$. But now if $i$ declares a preference $\bar{R}_{i}$ such that $G\left(\mathcal{O}, \bar{R}_{i}\right)=G\left(\mathcal{O}, R_{i}^{\prime}\right) \cup\{o\}$, the outcome $f\left(\bar{R}_{i}, R_{C \backslash\{i\}}^{\prime}, R_{N \backslash C}\right)=$ $f\left(R_{C}^{\prime}, R_{N \backslash C}\right) \cup\{o\}$, which is, by separability, strictly better for $i$ under $R_{i}$ than what she would get by following the agreed upon strategy. Therefore, no profitable deviation is credible under quota 1. A similar argument applies for quota $n$.

This already proves the proposition for the case $n=2$ since there only the two extreme quotas can be used. From now on we treat the case $n>3$.

To prove that any voting by quota rule $q, q \neq\{1, n\}$ violates immunity to credible deviations we construct profiles against which there is a credible profitable deviation by some coalition. We begin by the case $K=2$ and then argue that this can be embedded in a general profile presenting the same deviations whenever $K>2$.

Let $n$ be odd. We distinguish three subcases.

(1) $q>\frac{n-1}{2}+1$. Let $R_{N}$ be as follows: the preferences of any agent $i$ in a set of $\frac{n-1}{2}$ agents are such that $o_{1} P_{i}\left\{o_{1}, o_{2}\right\} P_{i} \varnothing$, the preferences of any agent $j$ in a different set of $\frac{n-1}{2}$ agents are such that $o_{2} P_{j}\left\{o_{1}, o_{2}\right\} P_{j} \varnothing$, and the preference of the remaining agent $l$ is such that $\tau\left(R_{l}\right)=\left\{o_{1}, o_{2}\right\}$. Observe that $f\left(R_{N}\right)=\varnothing$. Let $C$ be the coalition of all agents except agent $l$, let $R_{C}^{\prime}$ be such that each agent $i \in C, \tau\left(R_{i}^{\prime}\right)=\left\{o_{1}, o_{2}\right\}$. Observe that since $f\left(R_{C}^{\prime}, R_{N \backslash C}\right)=\left\{o_{1}, o_{2}\right\}, R_{C}^{\prime}$ is a profitable deviation of $C$ against $R_{N}$. Finally, $R_{C}^{\prime}$ is credible since no agent can change the outcome by a unilateral deviation since $n>3$.

(2) $q=\frac{n-1}{2}+1$. Let $R_{N}$ be as follows: the preferences of any agent $i$ in a set of $\frac{n-1}{2}$ agents are such that $o_{1} P_{i}\left\{o_{1}, o_{2}\right\} P_{i} \varnothing$, the preferences of any agent $j$ in a different set of 
$\frac{n-1}{2}$ agents are such that $o_{2} P_{j}\left\{o_{1}, o_{2}\right\} P_{j} \varnothing$, and the preference of the remaining agent $l$ is such that $\tau\left(R_{l}\right)=\varnothing$. Observe that $f\left(R_{N}\right)=\varnothing$. Let $C$ be the coalition of all agents except agent $l$, let $R_{C}^{\prime}$ be such that each agent $i \in C, \tau\left(R_{i}^{\prime}\right)=\left\{o_{1}, o_{2}\right\}$. Observe that since $f\left(R_{C}^{\prime}, R_{N \backslash C}\right)=\left\{o_{1}, o_{2}\right\}, R_{C}^{\prime}$ is a profitable deviation of $C$ against $R_{N}$. Finally, $R_{C}^{\prime}$ is credible since no agent can change the outcome by a unilateral deviation since $n>3$.

(3) $q<\frac{n-1}{2}+1$. Let $R_{N}$ be as follows: the preferences of any agent $i$ in a set of $\frac{n-1}{2}$ agents are such that $o_{1} P_{i} \varnothing P_{i}\left\{o_{1}, o_{2}\right\}$, the preferences of any agent $j$ in a different set of $\frac{n-1}{2}$ agents are such that $o_{2} P_{j} \varnothing P_{j}\left\{o_{1}, o_{2}\right\}$, and the preference of the remaining agent $l$ is such that $\tau\left(R_{l}\right)=\varnothing$. Observe that $f\left(R_{N}\right)=\left\{o_{1}, o_{2}\right\}$. Let $C$ be the coalition of all agents except agent $l$, let $R_{C}^{\prime}$ be such that each agent $i \in C, \tau\left(R_{i}^{\prime}\right)=\varnothing$. Observe that since $f\left(R_{C}^{\prime}, R_{N \backslash C}\right)=\varnothing$, $R_{C}^{\prime}$ is a profitable deviation of $C$ against $R_{N}$. Finally, $R_{C}^{\prime}$ is credible since no agent can change the outcome by a unilateral deviation since $n>3$.

Let $n$ be even. We distinguish two subcases.

(1) $q>\frac{n}{2}$. Let $R_{N}$ be as follows: the preferences of any agent $i$ in a set of $\frac{n}{2}$ agents are such that $o_{1} P_{i}\left\{o_{1}, o_{2}\right\} P_{i} \varnothing$, the preferences of any agent $j$ in a different set of $\frac{n}{2}$ agents are such that $o_{2} P_{j}\left\{o_{1}, o_{2}\right\} P_{j} \varnothing$. Observe that $f\left(R_{N}\right)=\varnothing$. Let $C$ be the coalition of all agents, let $R_{C}^{\prime}$ be such that each agent $i \in C, \tau\left(R_{i}^{\prime}\right)=\left\{o_{1}, o_{2}\right\}$. Observe that since $f\left(R_{C}^{\prime}, R_{N \backslash C}\right)=\left\{o_{1}, o_{2}\right\}$, $R_{C}^{\prime}$ is a profitable deviation of $C$ against $R_{N}$. Finally, $R_{C}^{\prime}$ is credible since no agent can change the outcome by a unilateral deviation.

(2) $q \leq \frac{n}{2}$. Let $R_{N}$ be as follows: the preferences of any agent $i$ in a set of $\frac{n}{2}$ agents are such that $o_{1} P_{i} \varnothing P_{i}\left\{o_{1}, o_{2}\right\}$, the preferences of any agent $j$ in a different set of $\frac{n}{2}$ agents are such that $o_{2} P_{j} \varnothing P_{j}\left\{o_{1}, o_{2}\right\}$. Observe that $f\left(R_{N}\right)=\left\{o_{1}, o_{2}\right\}$. Let $C$ be the coalition of all agents, let $R_{C}^{\prime}$ be such that each agent $i \in C, \tau\left(R_{i}^{\prime}\right)=\varnothing$. Observe that since $f\left(R_{C}^{\prime}, R_{N \backslash C}\right)=\varnothing, R_{C}^{\prime}$ is a profitable deviation of $C$ against $R_{N}$. Finally, $R_{C}^{\prime}$ is credible since no agent can change the outcome by a unilateral deviation.

This is easily extended to the case $K>2$ by considering profiles where agents preferences are like the ones described in each case above for objects 1 and 2 , while all the agents share exactly the same preferences concerning other objects for all cases analyzed (for example, $o_{k} \in G\left(\mathcal{O}, \widehat{R}_{i}\right)$ for each $o_{k} \in \mathcal{O} \backslash\left\{o_{1}, o_{2}\right\}$, each $i \in N$ and each individual preference $\widehat{R}_{i}$ used in the analyzed cases).

Our next proposition covers the case $n=3$, which is not contemplated by the previous one.

Proposition 2 When $n=3$ and $K=2$, any voting by quota rule is immune to credible deviations. When $n=3$ and $K \geq 3$, voting by quota 1 and 3 are the only voting by quota rules satisfying immunity to credible deviations.

Proof. Let $N=\{1,2,3\}$ and $K=2$. For voting by quotas 1 and 3 the same argument in Proposition 1 applies. Consider voting by quota 2. As already remarked in Barberà, Sonnenschein, and Zhou (1991) this rule is not only strategy-proof but also efficient. Thus the only coalitions with profitable deviations consist of two agents. Let $R_{N}, C=\{i, j\}$, and $R_{C}^{\prime}$ be a profitable deviation of $C$ against $R_{N}$.

To be a profitable deviation, observe that, by separability and voting by quota 2 , either (1) 
both candidates are chosen under $\left(R_{C}^{\prime}, R_{N \backslash C}\right)$ but none under $R_{N}$, or (2) no candidate is chosen under $\left(R_{C}^{\prime}, R_{N \backslash C}\right)$ but both are chosen under $R_{N}$, or (3) only one candidate is chosen under $R_{N}$ and only the other candidate is chosen under $\left(R_{C}^{\prime}, R_{N \backslash C}\right)$.

In the first case, for each candidate, one of the agents in $C$ considered that candidate not good under $R_{i}$ but good under $R_{i}^{\prime}$. In the second case, for each candidate, one of the agents in $C$ considered that candidate good under $R_{i}$ but not good under $R_{i}^{\prime}$. In the third case, what said in the second case holds for the candidate chosen under $R_{N}$ and what said in the first case holds for the candidate chosen under $\left(R_{C}^{\prime}, R_{N \backslash C}\right)$.

In the three cases, either declaring $\bar{R}_{i}$ such that a good candidate under $R_{i}^{\prime}$ not to be under $\bar{R}_{i}$, or supporting a bad one will be an individual profitable deviation with respect to $\left(R_{C}^{\prime}, R_{N \backslash C}\right)$. Thus, $R_{C}^{\prime}$ is not credible.

Let $N=\{1,2,3\}$ and $K=3$. For voting by quota 1 and 3 the same argument in Proposition 1 applies. To prove that voting by quota 2 violates immunity to credible deviations we provide an example of a credible profitable deviation against a profile. Let $R_{N}$ be as follows: the preferences of agent 1 are such that $\tau\left(R_{1}\right)=o_{1}$ and $\left\{o_{1}, o_{2}, o_{3}\right\} P_{1} \varnothing$, the preferences of agent 2 are such that $\tau\left(R_{2}\right)=o_{2}$ and $\left\{o_{1}, o_{2}, o_{3}\right\} P_{2} \varnothing$, and the preferences of agent 3 are such that $\tau\left(R_{3}\right)=o_{3}$ and $\left\{o_{1}, o_{2}, o_{3}\right\} P_{3} \varnothing$. Observe that $f\left(R_{N}\right)=\varnothing$. Let $C=N$, and $R_{N}^{\prime}$ be such that each agent $i \in C, \tau\left(R_{i}^{\prime}\right)=\left\{o_{1}, o_{2}, o_{3}\right\}$. Since $f\left(R_{N}^{\prime}\right)=\left\{o_{1}, o_{2}, o_{3}\right\}, R_{N}^{\prime}$ is a profitable deviation of $C$ against $R_{N}$. Finally, $R_{N}^{\prime}$ is credible since no agent can change the outcome by a unilateral deviation.

This is easily extended to the case $K>3$ by considering profiles where agents preferences are like the ones described in each case above for objects 1,2 , and 3 , while all the agents share exactly the same preferences concerning other objects for all cases analyzed (for example, $o_{k} \in G\left(\mathcal{O}, \widehat{R}_{i}\right)$ for each $o_{k} \in \mathcal{O} \backslash\left\{o_{1}, o_{2}, o_{3}\right\}$, each $i \in N$ and each individual preference $\widehat{R}_{i}$ used in the analyzed cases).

\subsection{The general case: choosing from a grid}

We now consider the general case where the set of possible choices in each dimension is not binary, as discussed in Barberà, Gul, and Stacchetti (1993). As we mentioned above, we consider $K \geq 2$ since for $K=1$ any strategy-proof rule is also group strategy-proof and thus immune to credible profitable deviations. The following three propositions allow us to identify the class of generalized Condorcet winner rules that are immune to credible deviations. Notice that Propositions 3 and 4 completely characterize the case with at least four agents in the society. Proposition 4 also covers the case of two agents. The case of three agents, requiring special treatment, is provided by Proposition 5 .

As we have already remarked, our analysis in the preceding subsection is a special case of what comes ahead. Let us then use some of the intuition we got from the previous analysis to anticipate the results that come. In the choice of sets example (with the necessary qualifications regarding number of voters and alternatives) the rules that emerge as not being vulnerable are those where unanimity is required to either impose each alternative, or to avoid it. In a similar spirit, we will see that, for functions that are not vulnerable in the general case there must be a specific privileged value in the interval corresponding to 
each dimension, and that only unanimous decisions against the prevalence of this particular value will avoid its selection. Hence, immunity to credible deviations requires a privileged treatment of one alternative per dimension, and a unanimity requirement to escape it.

Here are the Propositions.

Proposition 3 Let $n>3$. Let $f$ be a generalized Condorcet winner rule. If $f$ is defined by lists of parameters that are non-degenerate in at least two dimensions, then $f$ is not immune to credible deviations.

Proposition 4 Let $n \geq 2$. Let $f$ be a generalized Condorcet winner rule. If $f$ is defined by lists of parameters that are degenerate in at least $K-1$ dimensions, then $f$ is immune to credible deviations.

Proposition 5 Let $n=3$. Any generalized Condorcet winner rule defined by lists of parameters such that are non-degenerate in two dimensions is immune to credible deviations. Any generalized Condorcet winner rule defined by non-degenerate lists of parameters in at least three dimensions is not immune to credible deviations.

Note that for societies with two agents, generalized Condorcet winner rules have only one parameter, thus, since the list of parameters is degenerate in each dimension, Proposition 4 covers the 2-agents case. Moreover, although we only use the result in Proposition 4 for the cases $n=2$ and $n \geq 4$, we prove it for the 3 -agents case for completeness.

Now we turn to the proof of the above Propositions.

Proof of Proposition 3. Let $f$ be a generalized Condorcet winner rule with two dimensions, say 1 and 2, for which $P_{1}(f)$ and $P_{2}(f)$ are not degenerate. Consider the median(s), med $P_{1}(f)$ and med $P_{2}(f)$ of these parameters' lists. These medians may be unique or consist of two contiguous points, say med ${ }^{-} P_{k}(f)<$ med $^{+} P_{k}(f)$, for each $k \in\{1,2\}$.

In all cases below, in any profile we will define the preferences of each agent in $N$ concerning dimensions different from 1 and 2 to be the same and with top at some point $x_{k}$ in $B_{k}$, $k \in \mathcal{K} \backslash\{1,2\}$.

Assume first that for each $k \in\{1,2\}, \operatorname{med}^{-} P_{k}(f) \neq$ med $^{+} P_{k}(f)$. This can only happen if $n$ is odd and thus the number of parameters is even. Consider a partition of $N$ into three sets, $\bar{N}, \widetilde{N}$, and $l$ where $l$ is a singleton and such that $\# \bar{N}=\# \widetilde{N}$. Let the projections of $R_{N}$ in dimensions 1 and 2 be as follows. For agents in $\bar{N}$, let the $k$-dimensional top be $\operatorname{med}^{+} P_{k}(f)$ for $k \in\{1,2\}$. For agents in $\widetilde{N}$, let the $k$-dimensional top be $\operatorname{med}^{-} P_{k}(f)$ for $k \in\{1,2\}$. Agent $l$ has the $1-$ dimensional top at $m e d^{+} P_{1}(f)$ and the $2-$ dimensional top at med $^{-} P_{2}(f)$. Also assume for agents in $\bar{N} \cup \widetilde{N}$ that $\left(\right.$ med $^{-} P_{1}(f)$, med $\left.^{+} P_{2}(f), x_{\mathcal{K} \backslash\{1,2\}}\right) P_{i}\left(\right.$ med $^{+} P_{1}(f)$, med $\left.^{-} P_{2}(f), x_{\mathcal{K} \backslash\{1,2\}}\right)$. Observe that $f_{k}\left(R_{N}\right)=\tau_{k}\left(R_{l}\right)$ for each $k \in\{1,2\}$ and $f_{k}\left(R_{N}\right)=x_{k}$ for each $k \in \mathcal{K} \backslash\{1,2\}$. This is because, for each $k \in\{1,2\}, \tau_{k}\left(R_{l}\right)$ tie-breaks when computing $f_{k}$ as the median of all tops and parameters in $B_{k}$. Let $C=\bar{N} \cup \widetilde{N}$ and let $R_{C}^{\prime}$ be such that for each agent 
$i \in C, \tau_{1}\left(R_{i}^{\prime}\right)=$ med $^{-} P_{1}(f), \tau_{2}\left(R_{i}^{\prime}\right)=$ med $^{+} P_{2}(f) .{ }^{9}$ Observe that $f_{k}\left(R_{C}^{\prime}, R_{N \backslash C}\right)=\tau_{k}\left(R_{i}^{\prime}\right)$ for $k \in\{1,2\}$, and $f_{k}\left(R_{C}^{\prime}, R_{N \backslash C}\right)=x_{k}$ for each $k \in \mathcal{K} \backslash\{1,2\}$. This is because, for each $k, f_{k}\left(R_{C}^{\prime}, R_{N \backslash C}\right)$ is the top for individual preferences in $\left(R_{C}^{\prime}, R_{N \backslash C}\right)$ for $n-1$ agents and it coincides with med $^{-} P_{1}(f)$ in dimension 1 and with med $^{+} P_{2}(f)$ in dimension 2 . By definition, this shows that $R_{C}^{\prime}$ is a profitable deviation of $C$ against $R_{N}$.

Moreover, for each dimension $k \in\{1,2\}$, since $f_{k}\left(R_{C}^{\prime}, R_{N \backslash C}\right)$ is either med $^{-} P_{k}(f)$ or med $^{+} P_{k}(f)$ and, given that $n>3$, there are at least two parameters smaller or equal than $f_{k}\left(R_{C}^{\prime}, R_{N \backslash C}\right)=$ med $^{-} P_{k}(f)$ or greater or equal than $f_{k}\left(R_{C}^{\prime}, R_{N \backslash C}\right)=$ med $^{+} P_{k}(f)$.

Therefore, $f_{k}\left(R_{C}^{\prime}, R_{N \backslash C}\right)$ receives at least $n+1$ total votes for each $k \in\{1,2\}$. Hence, the profitable deviation $R_{C}^{\prime}$ is credible and $f$ is not immune to credible deviations.

Assume now that for at least some $k \in\{1,2\}, \operatorname{med}^{-} P_{k}(f)=\operatorname{med}^{+} P_{k}(f)=\operatorname{medP}_{k}(f)$.

Remember that med $^{-} P_{k}(f) \neq$ med $^{+} P_{k}(f)$ can only hold if $n$ is odd and therefore the number of parameters is even. Because of that in the case where the above equality holds for only one of the two dimensions but not for the other can only happen when $n$ is odd. This distinction is used along the rest of the proof because in one case a partition will only use two sets of agents $\bar{N}, \widetilde{N}$ while in other cases we will have to add a singleton $l$ to it.

For $n$ odd, let $\bar{N}, \widetilde{N}$, and agent $l$ be the elements of a partition of $N$ such that $\# \bar{N}=\# \widetilde{N}=$ $\frac{n-1}{2}$. For $n$ even, let, $\bar{N}, \widetilde{N}$ a partition of $N$ such that $\# \bar{N}=\# \widetilde{N}=\frac{n}{2}$. Let $R_{N}$ be as follows. The preferences of agents in $\bar{N}$ are such that in the dimension 1 the top is either ed $^{+} P_{1}(f)$ when med $^{-} P_{1}(f) \neq$ med $^{+} P_{1}(f)$, or med $P_{1}(f)$, otherwise. In dimension 2 the top is either med $^{+} P_{2}(f)$ when med $^{-} P_{2}(f) \neq$ med $^{+} P_{2}(f)$, or the highest parameter strictly smaller than med $P_{2}(f)$ if it exists and med $^{-} P_{2}(f)=$ med $^{+} P_{2}(f)$, or the lowest parameter strictly greater than medP $P_{2}(f)$, otherwise. The preferences of agents in $\tilde{N}$ are such that in dimension 1 the top is either med $^{-} P_{1}(f)$ when med $^{-} P_{1}(f) \neq$ med $^{+} P_{1}(f)$, or the highest parameter strictly smaller than $\operatorname{med} P_{1}(f)$ if it exists and med $^{-} P_{1}(f)=$ med $^{+} P_{1}(f)$, or the lowest parameter strictly greater than med $P_{1}(f)$, otherwise. In dimension 2 the top is either med $^{-} P_{2}(f)$ when med $^{-} P_{2}(f) \neq$ med $^{+} P_{2}(f)$, or med $P_{2}(f)$, otherwise.

Preferences of agent $l$ (only required if $n$ is odd) are defined as follows: $R_{l}$ is such that in dimension 1 agent l's top is either $\tau_{1}\left(R_{j}\right), j \in \bar{N}$ when med $^{-} P_{1}(f) \neq$ med $^{+} P_{1}(f)$, or the highest parameter strictly smaller than med $P_{1}(f)$ if such parameter exists or the lowest parameter strictly greater than med $P_{1}(f)$, otherwise. In dimension 2 the top of agent $l$ is either either $\tau_{2}\left(R_{i}\right), i \in \widetilde{N}$ when med $^{-} P_{2}(f) \neq$ med $^{+} P_{2}(f)$, or the highest parameter strictly smaller than med $P_{2}(f)$ if such parameter exists or the lowest parameter strictly greater than med $P_{2}(f)$, otherwise.

From now on let $i \in \widetilde{N}$ and $j \in \bar{N}$. We also assume that for any agent $m \in \bar{N} \cup$ $\widetilde{N},\left(\tau_{1}\left(R_{i}\right), \tau_{2}\left(R_{j}\right), x_{\mathcal{K} \backslash\{1,2\}}\right) P_{m}\left(\tau_{1}\left(R_{j}\right), \tau_{2}\left(R_{i}\right), x_{\mathcal{K} \backslash\{1,2\}}\right)$. Observe that $f_{k}\left(R_{N}\right)=\tau_{k}\left(R_{l}\right)$ if med $^{-} P_{k}(f) \neq$ med $^{+} P_{k}(f)$, and $f_{k}\left(R_{N}\right)=\operatorname{med}_{k}(f)$ otherwise for each $k \in\{1,2\}$, and that $f_{k}\left(R_{N}\right)=x_{k}$ for each $k \in \mathcal{K} \backslash\{1,2\}$. This is because, for each $k \in\{1,2\}, \tau_{k}\left(R_{l}\right)$ tie-breaks when computing $f_{k}$ as the median of all tops and parameters in $B_{k}$ in the case where only for one $k \in\{1,2\}$, med ${ }^{-} P_{k}(f)=$ med $^{+} P_{k}(f)=\operatorname{med}_{k}(f)$ and thus $n$ is odd. And for each

\footnotetext{
${ }^{9}$ In words, to define $R_{C}^{\prime}$ notice that by changing their vote the agents in $\bar{N}$ vote for the tops of those in $\widetilde{N}$ in dimension 1, while agents in $\widetilde{N}$ vote for the top of those in $\bar{N}$ in dimension 2 .
} 
$k \in\{1,2\}, \operatorname{med} P_{k}(f)$ tie-breaks when computing $f_{k}$ as the median of all tops and parameters in $B_{k}$ in the case where for both $k \in\{1,2\}, \operatorname{med}^{-} P_{k}(f)=\operatorname{med}^{+} P_{k}(f)=\operatorname{med}_{k}(f)$. Let $C=$ $\bar{N} \cup \widetilde{N}$ and let $R_{C}^{\prime}$ be such that for each agent $j \in \bar{N}, \tau_{1}\left(R_{j}^{\prime}\right)=\tau_{1}\left(R_{i}\right)$ and $\tau_{k}\left(R_{j}^{\prime}\right)=\tau_{k}\left(R_{j}\right)$ for each $k \in \mathcal{K} \backslash\{1\}$, and for each $i \in \widetilde{N}, \tau_{2}\left(R_{i}^{\prime}\right)=\tau_{2}\left(R_{j}\right)$ and $\tau_{k}\left(R_{i}^{\prime}\right)=\tau_{k}\left(R_{i}\right)$ for each $k \in \mathcal{K} \backslash\{2\}$. Observe that $f_{k}\left(R_{C}^{\prime}, R_{N \backslash C}\right)=\tau_{k}\left(R_{i}^{\prime}\right)$ for $k \in\{1,2\}$, and $f_{k}\left(R_{C}^{\prime}, R_{N \backslash C}\right)=x_{k}$ for each $k \in \mathcal{K} \backslash\{1,2\}$. This is because, for each $k$ where med $^{-} P_{k}(f)=\operatorname{med}^{+} P_{k}(f)$, $f_{k}\left(R_{C}^{\prime}, R_{N \backslash C}\right)$ is the top for individual preferences in $\left(R_{C}^{\prime}, R_{N \backslash C}\right)$ for $n$ agents. For each $k$ where $\operatorname{med}^{-} P_{k}(f) \neq$ med $^{+} P_{k}(f), f_{k}\left(R_{C}^{\prime}, R_{N \backslash C}\right)$ is the top for the preferences for $n-1$ agents in $\left(R_{C}^{\prime}, R_{N \backslash C}\right)$ and coincides either with $\operatorname{med}^{-} P_{k}(f)$ or $\operatorname{med}^{+} P_{k}(f)$. By definition, this shows that $R_{C}^{\prime}$ is a profitable deviation of $C$ against $R_{N}$.

Moreover, for the dimensions where $\operatorname{med}^{-} P_{k}(f)=\operatorname{med}^{+} P_{k}(f)$ there is a parameter at $f_{k}\left(R_{C}^{\prime}, R_{N \backslash C}\right)$. For the dimensions where med $^{-} P_{k}(f) \neq$ med $^{+} P_{k}(f), f_{k}\left(R_{C}^{\prime}, R_{N \backslash C}\right)$ is either med $^{-} P_{k}(f)$ or med $^{+} P_{k}(f)$ and, given that $n>3$, there are at least two parameters smaller or equal than $f_{k}\left(R_{C}^{\prime}, R_{N \backslash C}\right)=$ med $^{-} P_{k}(f)$ or greater or equal than $f_{k}\left(R_{C}^{\prime}, R_{N \backslash C}\right)=$ med $^{+} P_{k}(f)$. Therefore, $f_{k}\left(R_{C}^{\prime}, R_{N \backslash C}\right)$ receives at least $n+1$ total votes for each $k \in\{1,2\}$. Hence, the profitable deviation $R_{C}^{\prime}$ is credible and $f$ is not immune to credible deviations.

Before we prove Propositions 4 and 5, we need some definitions, claims and lemmas.

Let $\mathcal{S}_{B_{k}}$ be the set of all unidimensional (strict) single-peaked preferences on $B_{k}$ (for a formal definition see, for example, Definition 6 when $B$ is an integer interval).

Definition 10 Let $f$ be a generalized Condorcet winner rule. For any $k \in \mathcal{K}$, define $F_{k}$ : $\left(\mathcal{S}_{B_{k}}\right)^{n} \rightarrow B_{k}$ such that for any $\widetilde{R}_{N} \in\left(\mathcal{S}_{B_{k}}\right)^{n}, F_{k}\left(\widetilde{R}_{N}\right)=f_{k}\left(R_{N}\right)$ for any $R_{N} \in \mathcal{S}^{n}$ such that $\tau_{k}\left(R_{i}\right)=\tau\left(\widetilde{R}_{i}\right)$ for any $i \in N$.

Note that $F_{k}$ is well-defined since $f_{k}$ is tops-only and any $R_{N}$ as defined will work. Moreover, $F_{k}$ is a unidimensional generalized Condorcet winner rule as in Definition 7.

Definition 11 Let $f$ be a generalized Condorcet winner rule, $R_{C}^{\prime}$ be a profitable deviation of $C$ against $R_{N}$ for $f$ and let $k \in \mathcal{K}$ such that $f_{k}\left(R_{C}^{\prime}, R_{N \backslash C}\right) \neq f_{k}\left(R_{N}\right)$. We say that agent $i \in C$ is losing according to $R_{i}$ at $\left(R_{C}^{\prime}, R_{N \backslash C}\right)$ in dimension $k \in \mathcal{K}$ if

$$
\left(f_{k}\left(R_{N}\right), f_{\mathcal{K} \backslash\{k\}}\left(R_{C}^{\prime}, R_{N \backslash C}\right)\right) P_{i} f\left(R_{C}^{\prime}, R_{N \backslash C}\right) .
$$

Define $L_{k}\left(f, R_{C^{\prime}}, R_{N}\right)=\left\{i \in C: i\right.$ is losing according to $R_{i}$ at $\left(R_{C}^{\prime}, R_{N \backslash C}\right)$ in dimension $\left.k \in \mathcal{K}\right\}$. Similarly, we say that agent $i \in C$ is winning according to $R_{i}$ at $\left(R_{C}^{\prime}, R_{N \backslash C}\right)$ in dimension $k \in \mathcal{K}$ if

$$
f\left(R_{C}^{\prime}, R_{N \backslash C}\right) P_{i}\left(f_{k}\left(R_{N}\right), f_{\mathcal{K} \backslash\{k\}}\left(R_{C}^{\prime}, R_{N \backslash C}\right)\right) .
$$

Define $W_{k}\left(f, R_{C^{\prime}}, R_{N}\right)=\left\{i \in C: i\right.$ is winning according to $R_{i}$ at $\left(R_{C}^{\prime}, R_{N \backslash C}\right)$ in dimension $\left.k \in \mathcal{K}\right\}$.

In short, when no confusion may arise, we will say that $i$ is losing or winning in dimension $k$ and we denote the sets of losers and winners as $L_{k}$ and $W_{k}$, respectively. Note that $L_{k}$ and $W_{k}$ is a partition of $C$ by definition since preferences are strict. That is, $L_{k} \cup W_{k}=C$ and $L_{k} \cap W_{k}=\varnothing$. 
Claim 1 Let $i \in C \subseteq N, R_{i} \in \mathcal{S}, x \in B$. Define $\widehat{R}_{i} \in \mathcal{S}$ such that (1) $\tau\left(\widehat{R}_{i}\right)=\tau\left(R_{i}\right)$, (2) for any $k \in \mathcal{K}$, for any $z_{k}, w_{k} \in B_{k}$, for $x_{\mathcal{K} \backslash\{k\}}$,

$$
\left(z_{k}, x_{\mathcal{K} \backslash\{k\}}\right) \widehat{P}_{i}\left(w_{k}, x_{\mathcal{K} \backslash\{k\}}\right) \Leftrightarrow\left(z_{k}, x_{\mathcal{K} \backslash\{k\}}\right) P_{i}\left(w_{k}, x_{\mathcal{K} \backslash\{k\}}\right) \text {, and }
$$

(3) for any $k \in \mathcal{K}$, for any $z_{\mathcal{K} \backslash\{k\}}, w_{\mathcal{K} \backslash\{k\}} \in B_{\mathcal{K} \backslash\{k\}}$, for any $y_{k}, v_{k} \in B_{k}$,

$$
\left(y_{k}, z_{\mathcal{K} \backslash\{k\}}\right) \widehat{P}_{i}\left(v_{k}, z_{\mathcal{K} \backslash\{k\}}\right) \Leftrightarrow\left(y_{k}, w_{\mathcal{K} \backslash\{k\}}\right) \widehat{P}_{i}\left(v_{k}, w_{\mathcal{K} \backslash\{k\}}\right) .
$$

We leave the proof to the reader. Note however, that $\widehat{R}_{i}$ exists and additively representable separable preferences work. A separable preference (as defined in Le Breton and Sen, 1999) induces the same ordering over dimension $j$ for any alternative. Claim 1 says that starting from any preference relation $R$ and any alternative $x$, we can construct a separable preference where the ordering in each dimension is the one induced by $R$ over that dimension relative to alternative $x$.

Claim 2 Let $f$ be a generalized Condorcet winner rule and let $R_{C}^{\prime}$ be a profitable deviation of $C$ against $R_{N}$ for $f$. Then:

(i) For any agent $i \in C$, there exists $k \in \mathcal{K}$ for which $f_{k}\left(R_{C}^{\prime}, R_{N \mid C}\right) \neq f_{k}\left(R_{N}\right)$ such that $f\left(R_{C}^{\prime}, R_{N \backslash C}\right) P_{i}\left(f_{k}\left(R_{N}\right), f_{\mathcal{K} \backslash\{k\}}\left(R_{C}^{\prime}, R_{N \backslash C}\right)\right)$.

(ii) If agent $i \in C$ is such that $f\left(R_{C}^{\prime}, R_{N \backslash C}\right) P_{i}\left(f_{k}\left(R_{N}\right), f_{\mathcal{K} \backslash\{k\}}\left(R_{C}^{\prime}, R_{N \backslash C}\right)\right)$ for some $k$, then there exists another agent $j \in C$ such that $\left(f_{k}\left(R_{N}\right), f_{\mathcal{K} \backslash\{k\}}\left(R_{C}^{\prime}, R_{N \backslash C}\right)\right) P_{j} f\left(R_{C}^{\prime}, R_{N \backslash C}\right)$.

(iii) For any $k \in \mathcal{K}$, there $i$ s an agent $i \in C$ such that $\left(f_{k}\left(R_{N}\right), f_{\mathcal{K} \backslash\{k\}}\left(R_{C}^{\prime}, R_{N \backslash C}\right)\right) P_{i} f\left(R_{C}^{\prime}, R_{N \backslash C}\right)$.

In words: (i) any agent in the profitable deviating coalition is winning in some dimension, (ii) if an agent in the profitable deviating coalition is winning in some dimension, there is another agent in the deviating coalition losing in the same dimension. Part (iii) ensures that there is at least a losing agent in each dimension.

We now turn to stating Lemmas 2 and 3.

Lemma 2 Let $f$ be a generalized Condorcet winner rule and let $R_{C}^{\prime}$ be a profitable deviation of $C$ against $R_{N}$ for $f$. There exist $k, k^{\prime} \in \mathcal{K}$ and $i, j \in C$ such that the following holds: $\left(f_{k}\left(R_{N}\right), f_{\mathcal{K} \backslash\{k\}}\left(R_{C}^{\prime}, R_{N \backslash C}\right)\right) P_{i} f\left(R_{C}^{\prime}, R_{N \backslash C}\right),\left(f_{k^{\prime}}\left(R_{N}\right), f_{\mathcal{K} \backslash\left\{k^{\prime}\right\}}\left(R_{C}^{\prime}, R_{N \backslash C}\right)\right) P_{j} f\left(R_{C}^{\prime}, R_{N \backslash C}\right)$, $\left(f_{k^{\prime}}\left(R_{C}^{\prime}, R_{N \backslash C}\right), f_{\mathcal{K} \backslash\left\{k^{\prime}\right\}}\left(R_{C}^{\prime}, R_{N \backslash C}\right)\right) P_{i}\left(f_{k^{\prime}}\left(R_{N}\right), f_{\mathcal{K} \backslash\left\{k^{\prime}\right\}}\left(R_{C}^{\prime}, R_{N \backslash C}\right)\right)$, and $\left(f_{k}\left(R_{C}^{\prime}, R_{N \backslash C}\right), f_{\mathcal{K} \backslash\{k\}}\left(R_{C}^{\prime}, R_{N \backslash C}\right)\right) P_{j}\left(f_{k}\left(R_{N}\right), f_{\mathcal{K} \backslash\{k\}}\left(R_{C}^{\prime}, R_{N \backslash C}\right)\right)$.

Lemma 2 formalizes the idea that in any profitable deviation there exist two agents and two dimensions where agents are exchanging roles and helping each other.

Lemma 3 Let $f$ be a generalized Condorcet winner rule, $R_{C}^{\prime}$ be a profitable deviation of $C$ against $R_{N}$ for $f$ and $k$ be such that $P_{k}(f)$ is degenerate. If there exists $i \in C$ winning at $\left(R_{C}^{\prime}, R_{N \backslash C}\right)$ in dimension $k$, then, the profitable deviation $R_{C}^{\prime}$ is not credible. 
Lemma 3 says that a credible profitable deviation relative to a generalized Condorcet winner rule cannot involve an agent winning in a degenerate dimension.

The proofs of Claim 2, and those of Lemmas 2 and 3 are in the Appendix. Finally, we can provide a proof for Propositions 4 and 5.

Proof of Proposition 4. Consider $f$ as in the statement. By contradiction, let $R_{N} \in \mathcal{S}^{n}$, $C \subseteq N$, and $R_{C}^{\prime} \in \mathcal{S}^{c}$ be a profitable deviation of $C$ against $R_{N}$. By Lemma 2, there exist $k, k^{\prime} \in \mathcal{K}$ and $i, j \in C$ such that

$$
\begin{gathered}
\left(f_{k}\left(R_{N}\right), f_{\mathcal{K} \backslash\{k\}}\left(R_{C}^{\prime}, R_{N \backslash C}\right)\right) P_{i}\left(f_{k}\left(R_{C}^{\prime}, R_{N \backslash C}\right), f_{\mathcal{K} \backslash\{k\}}\left(R_{C}^{\prime}, R_{N \backslash C}\right)\right), \\
\left(f_{k}\left(R_{C}^{\prime}, R_{N \backslash C}\right), f_{\mathcal{K} \backslash\{k\}}\left(R_{C}^{\prime}, R_{N \backslash C}\right)\right) P_{j}\left(f_{k}\left(R_{N}\right), f_{\mathcal{K} \backslash\{k\}}\left(R_{C}^{\prime}, R_{N \backslash C}\right)\right), \\
\left(f_{k^{\prime}}\left(R_{C}^{\prime}, R_{N \backslash C}\right), f_{\mathcal{K} \backslash\left\{k^{\prime}\right\}}\left(R_{C}^{\prime}, R_{N \backslash C}\right)\right) P_{i}\left(f_{k^{\prime}}\left(R_{N}\right), f_{\mathcal{K} \backslash\left\{k^{\prime}\right\}}\left(R_{C}^{\prime}, R_{N \backslash C}\right)\right), \text { and } \\
\left(f_{k^{\prime}}\left(R_{N}\right), f_{\mathcal{K} \backslash\left\{k^{\prime}\right\}}\left(R_{C}^{\prime}, R_{N \backslash C}\right)\right) P_{j}\left(f_{k^{\prime}}\left(R_{C}^{\prime}, R_{N \backslash C}\right), f_{\mathcal{K} \backslash\left\{k^{\prime}\right\}}\left(R_{C}^{\prime}, R_{N \backslash C}\right)\right) .
\end{gathered}
$$

By hypothesis, either $P_{k}(f)$ or $P_{k^{\prime}}(f)$ is degenerate (or both). ${ }^{10}$ By Lemma $3, R_{C}^{\prime}$ is not a credible profitable deviation. This ends the proof.

Proof of Proposition 5. To prove the first statement, let $f$ be a generalized Condorcet winner rule with lists of parameters, denoted in each dimension $k$ as $p_{k}^{-} \leq p_{k}^{+}$, and such that they are non-degenerate in exactly two dimensions. To prove that $f$ is immune to credible deviations, let $R_{N} \in \mathcal{S}^{3}, C \subseteq N=\{1,2,3\}$, and $R_{C}^{\prime} \in \mathcal{S}^{c}$ be a profitable deviation of $C$ against $R_{N}$. If the profitable deviation is such that there is an agent that is winning in a dimension $k$ for which $P_{k}(f)$ is degenerate. Thus, by Lemma $3, R_{C}^{\prime}$ would not be a credible profitable deviation. Now assume that agents are winning at $\left(R_{C}^{\prime}, R_{N \backslash C}\right)$ in dimensions $k$ for which $P_{k}(f)$ is not degenerate. By Lemma 2, there must be agents in $C$ winning at $\left(R_{C}^{\prime}, R_{N \backslash C}\right)$ in the two dimensions with non-degenerate list of parameters $P_{k}(f)$. Without loss of generality, assume that these dimensions are 1 and 2. By strategy-proofness, $C$ has at least two agents. Without loss of generality, by anonymity and Lemma 2, suppose that agents 1 and 2 belong to $C$ and that agent 1 is winning while agent 2 is losing at $\left(R_{C}^{\prime}, R_{N \backslash C}\right)$ in dimension 1 and the opposite holds in dimension 2. Consider the following two cases depending on the size of the deviating coalition.

Case 1: $C=\{1,2\}$.

Since agent 3's preferences are fixed, we can assume now that we have 3 fixed parameters in each dimension, two of them different: $p_{k}^{-}, p_{k}^{+}$, and $\tau\left(R_{3}\right)$. Consider dimension 1. First, observe that

$$
f_{1}\left(R_{N}\right), f_{1}\left(R_{\{1,2\}}^{\prime}, R_{3}\right) \in\left[\min \left\{\tau_{1}\left(R_{3}\right), p_{1}^{-}\right\}, \max \left\{\tau_{1}\left(R_{3}\right), p_{1}^{+}\right\}\right] .
$$

Since agent 1 is winning and agent 2 is losing at $\left(R_{\{1,2\}}^{\prime}, R_{3}\right)$ in dimension 1 , then $\tau_{1}\left(R_{1}\right)$ must be strictly placed on one side of $f_{1}\left(R_{N}\right)$, while $\tau_{1}\left(R_{2}\right)$ must be weakly placed on the other side of $f_{1}\left(R_{N}\right)$. Moreover, for both $i \in\{1,2\}, \tau_{1}\left(R_{i}^{\prime}\right)$ must be strictly in the same side of $f_{1}\left(R_{N}\right)$ and in fact in the same side as $\tau_{1}\left(R_{1}\right)$ is. Then, note that agent 2 announcing $\bar{R}_{2}$ such that

\footnotetext{
${ }^{10}$ For $n=2$ any list of parameters is degenerate in all dimensions since all parameters take the same value.
} 
$\tau_{1}\left(\bar{R}_{2}\right)=\tau_{1}\left(R_{2}\right)$ and for each $k \in \mathcal{K} \backslash\{1\}, \tau_{k}\left(\bar{R}_{2}\right)=\tau_{k}\left(R_{2}^{\prime}\right)$ would be winning at $\left(\bar{R}_{2}, R_{1}^{\prime}, R_{3}\right)$ in dimension 1 and by separability she would be better off $f\left(\bar{R}_{2}, R_{1}^{\prime}, R_{3}\right) P_{2} f\left(R_{\{1,2\}}^{\prime}, R_{3}\right)$ which means that $R_{\{1,2\}}^{\prime}$ is not a credible profitable deviation.

Case 2: $C=\{1,2,3\}$.

Since $R_{N}^{\prime} \in \mathcal{S}^{n}$ is a profitable deviation of $C$ against $R_{N}$, agent 3 is winning at $R_{N}^{\prime}$ in some dimension. Without loss of generality, suppose that agent 3 is winning at $R_{N}^{\prime}$ in dimension 1 (otherwise, by anonymity a similar argument would apply). We already assumed that agent 1 is winning and agent 2 is losing at $R_{N}^{\prime}$ in dimension 1 . If $f_{1}\left(R_{N}\right) \in\left(p_{1}^{-}, p_{1}^{+}\right)$, then $\tau_{1}\left(R_{2}\right)=f_{1}\left(R_{N}\right)$ since agent 2 is losing at $R_{N}^{\prime}$, and also both $\tau_{1}\left(R_{1}\right)$ and $\tau_{1}\left(R_{3}\right)$ must be strictly on a different side of $f_{1}\left(R_{N}\right)$. But then, $R_{C}^{\prime}$ would not be a profitable deviation where agents 1 and 3 are winning at $R_{N}^{\prime}$ in dimension 1 . Thus, $f_{1}\left(R_{N}\right) \leq p_{1}^{-}$ or $f_{1}\left(R_{N}\right) \geq p_{1}^{+}$. Suppose $f_{1}\left(R_{N}\right) \leq p_{1}^{-}$(a symmetric argument would apply for the other case). Observe first that for each $i \in\{1,2,3\}, \tau_{1}\left(R_{i}\right) \leq f_{1}\left(R_{N}\right)$. Since agent 2 is the only agent losing at $R_{N}^{\prime}$ in dimension $1, \tau_{1}\left(R_{2}\right)=f_{1}\left(R_{N}\right)$ and $\tau_{1}\left(R_{1}\right), \tau_{1}\left(R_{3}\right)<f_{1}\left(R_{N}\right)$, and thus $f_{1}\left(R_{C}^{\prime}, R_{N \backslash C}\right)<f_{1}\left(R_{N}\right)$. Then, note that agent 2 announcing $\bar{R}_{2}$ such that $\tau_{1}\left(\bar{R}_{2}\right)=\tau_{1}\left(R_{2}\right)$ and for each $k \in \mathcal{K} \backslash\{1\}, \tau_{k}\left(\bar{R}_{2}\right)=\tau_{k}\left(R_{2}^{\prime}\right)$ would be winning at $\left(\bar{R}_{2}, R_{\{1,3\}}^{\prime}\right)$ in dimension 1 and by separability she would be better off $f\left(\bar{R}_{2}, R_{\{1,3\}}^{\prime}\right) P_{2} f\left(R_{\{1,2,3\}}^{\prime}\right)$ which means that $R_{\{1,2,3\}}^{\prime}$ is not a credible profitable deviation.

For all cases we obtain that the profitable deviation is not credible which shows the first statement in the proposition.

To prove the second statement, let $f$ be a generalized Condorcet winner rule with lists of parameters, denoted in each dimension $k$ as $p_{k}^{-} \leq p_{k}^{+}$, and such that they are non-degenerate in at least three dimensions. Assume they are dimensions 1, 2, and 3. To prove that $f$ is not immune to credible deviations, we provide an example of a credible profitable deviation against a profile. In any profile we will define the preferences of each agent in $N$ concerning dimensions different from 1,2 , and 3 to be the same and with top at some point $x_{k}$ in $B_{k}$, $k \in \mathcal{K} \backslash\{1,2,3\}$.

Let $R_{N} \in \mathcal{S}^{3}$ be as follows in dimensions 1, 2, and 3: define the preferences of agent 1 such that $\tau\left(R_{1}\right)=\left(p_{1}^{+}, p_{2}^{-}, p_{3}^{-}\right)$and $\left(p_{1}^{+}, p_{2}^{+}, p_{3}^{+}, x_{\mathcal{K} \backslash\{1,2,3\}}\right) P_{1}\left(p_{1}^{-}, p_{2}^{-}, p_{3}^{-}, x_{\mathcal{K} \backslash\{1,2,3\}}\right)$, the preferences of agent 2 such that $\tau\left(R_{2}\right)=\left(p_{1}^{-}, p_{2}^{+}, p_{3}^{-}\right)$and $\left(p_{1}^{+}, p_{2}^{+}, p_{3}^{+}, x_{\mathcal{K} \backslash\{1,2,3\}}\right) P_{2}\left(p_{1}^{-}, p_{2}^{-}, p_{3}^{-}, x_{\mathcal{K} \backslash\{1,2,3\}}\right)$, and the preferences of agent 3 such that $\tau\left(R_{3}\right)=\left(p_{1}^{-}, p_{2}^{-}, p_{3}^{+}\right)$and $\left(p_{1}^{+}, p_{2}^{+}, p_{3}^{+}, x_{\mathcal{K} \backslash\{1,2,3\}}\right) P_{3}$ $\left(p_{1}^{-}, p_{2}^{-}, p_{3}^{-}, x_{\mathcal{K} \backslash\{1,2,3\}}\right)$. Observe that $f\left(R_{N}\right)=\left(p_{1}^{-}, p_{2}^{-}, p_{3}^{-}, x_{\mathcal{K} \backslash\{1,2,3\}}\right)$. Let $C=N$, and $R_{N}^{\prime}$ such that each agent $i \in C, \tau\left(R_{i}^{\prime}\right)=\left(p_{1}^{+}, p_{2}^{+}, p_{3}^{+}\right)$. Since $f\left(R_{N}^{\prime}\right)=\left(p_{1}^{+}, p_{2}^{+}, p_{3}^{+}\right), R_{N}^{\prime}$ is a profitable deviation of $C$ against $R_{N}$. Finally, $R_{N}^{\prime}$ is credible since no agent can change the outcome by a unilateral deviation.

This ends the proof.

\section{Some alternative formulations of credibility, and their consequences}

We believe that our definition of a credible deviation is quite attractive. But others could be conceivable, and in this section we shall discuss other possible proposals, and relate them 
to ours.

We could take several directions to obtain alternative definitions of immunity. First, we concentrate on varying the notion of credibility to which we devote more attention.

To favor the comparison, let us go back to the interpretation of credibility that we already proposed after Definition 4. A profitable deviation by $C$ from $R_{N}=\left(R_{C}, R_{N \backslash C}\right)$ is credible if $R_{C}^{\prime}$ is a Nash equilibrium of the game among agents in $C$, when these agents strategies are their admissible preferences and the outcome function is $f\left(\cdot, R_{N \backslash C}\right)$. Starting from this, we shall discuss, then, three possible variants of the credibility concept.

The first variant will be one where, instead of letting agents in $C$ to have any choice of preferences as a strategy, we restrict them to either use strategy $R_{i}^{\prime}$ or to revert to strategy $R_{i}$. The resulting notion of a credible deviation will be weaker than ours. However, we will show that the set of rules that are immune to credible deviations will be the same (after a minimal qualification) under either definition. This is expressed in Proposition 6.

A second variant will require that in order to be (extensively) credible, the deviation $R_{C}^{\prime}$ should be a Nash equilibrium for the game where all agents (whether or not they are part of $C$ ) can play any preference, and $f$ is the outcome function. If the initial function $f$ is assumed to be strategy-proof (an assumption that we do not need under our original definition), then again the set of rules immune to credible deviations will still be the same under either definition (see Proposition 7). However, the equivalence is not true if our $f$ function is not a priory restricted to be strategy-proof, as shown in Example 3.

A third variant of our definition of credibility would result from simply changing our original one, but ask the deviation to be a strong Nash, rather than a Nash equilibrium. The rationale for such proposal would be to allow for several agents to coordinate when defecting from the agreed upon joint manipulation. We will show that under this definition, all of the rules we consider will be immune to credible deviations (see Proposition 8). ${ }^{11}$

We now present formal arguments to make the preceding discussion more precise. We also state some results whose proofs are included in Appendix.

Definition 12 Let $f$ be a social choice function on $\mathcal{U}^{n}$. Let $R_{N} \in \mathcal{U}^{n}$ and $C \subseteq N$. We say that $R_{C}^{\prime} \in \mathcal{U}^{c}$ a profitable deviation of $C$ against $R_{N}$ is (type 1) credible if $f\left(R_{C}^{\prime}, R_{N \mid C}\right) R_{i} f\left(R_{i}, R_{C \backslash\{i\}}^{\prime}, R_{N \backslash C}\right)$ for all $i \in C$. A social choice function $f$ on $\mathcal{U}^{n}$ is immune to (type 1) credible deviations if for any $R_{N} \in \mathcal{U}^{n}$, any $C \subseteq N$, there is no (type 1) credible profitable deviation of $C$ against $R_{N}$.

Proposition 6 Any social choice function $f$ on $\mathcal{U}^{n}$ is immune to credible deviations if and only if $f$ is immune to (type 1) credible deviations.

Definition 13 Let $f$ be a social choice function on $\mathcal{U}^{n}$. Let $R_{N} \in \mathcal{U}^{n}$ and $C \subseteq N$. We say that $R_{C}^{\prime} \in \mathcal{U}^{c}$ a profitable deviation of $C$ against $R_{N}$ is (type 2) credible if $f\left(R_{C}^{\prime}, R_{N \mid C}\right) R_{i} f\left(\bar{R}_{i}, R_{C \backslash\{i\}}^{\prime}, R_{N \backslash(C \cup\{i\})}\right)$ for all $i \in N$ and all $\bar{R}_{i} \in \mathcal{U}$. A social choice function $f$ on $\mathcal{U}^{n}$ is immune to (type 2) credible deviations if for any $R_{N} \in \mathcal{U}^{n}$, any $C \subseteq N$, there is no (type 2) credible profitable deviation of $C$ against $R_{N}$.

\footnotetext{
${ }^{11}$ The same will hold if instead allowing agents to use any preferences, they are only assumed to use their true and the manipulative one.
} 
Proposition 7 Any strategy-proof social choice function $f$ on $\mathcal{U}^{n}$ is immune to credible deviations if and only if $f$ is immune to (type 2) credible deviations.

The following example shows that the latter immunity concept does not imply strategyproofness. Therefore, the concept may be useful to apply in contexts where strategyproofness is not to be expected, but one may still be interested in discussing the diversity of manipulative actions by groups of voters.

Example 3 Immunity to (type 2) credible deviations does not imply strategy-proofness under appropriately restricted domains. ${ }^{12}$

Let $K=2, N=\{1,2\}$, and for $i \in N$, let the set of admissible preferences for both agents be $\mathcal{S}$, that is, preferences defined as in Table 2.

\begin{tabular}{|c|c|c|c|c|c|c|c|}
\hline$R^{1}$ & $R^{2}$ & $R^{3}$ & $R^{4}$ & $R^{1^{\prime}}$ & $R^{2^{\prime}}$ & $R^{3^{\prime}}$ & $R^{4^{\prime}}$ \\
\hline$\varnothing$ & $o_{1}$ & $o_{1}$ & $\left\{o_{1}, o_{2}\right\}$ & $\varnothing$ & $o_{2}$ & $o_{2}$ & $\left\{o_{1}, o_{2}\right\}$ \\
\hline$o_{1}$ & $\varnothing$ & $\left\{o_{1}, o_{2}\right\}$ & $o_{1}$ & $o_{2}$ & $\varnothing$ & $\left\{o_{1}, o_{2}\right\}$ & $O_{2}$ \\
\hline$O_{2}$ & $\left\{o_{1}, o_{2}\right\}$ & $\varnothing$ & $O_{2}$ & $o_{1}$ & $\left\{o_{1}, o_{2}\right\}$ & $\varnothing$ & $O_{1}$ \\
\hline$\left\{o_{1}, o_{2}\right\}$ & $o_{2}$ & $o_{2}$ & $\varnothing$ & $\left\{o_{1}, o_{2}\right\}$ & $o_{1}$ & $o_{1}$ & $\varnothing$ \\
\hline
\end{tabular}

Table 2. The set $\mathcal{S}$ of all separable preferences when $K=2$.

Consider the social choice function $f$ defined as in Table 3.

\begin{tabular}{|c|c|c|c|c|}
\hline$f$ & $R_{2}^{1^{\prime}}, R_{2}^{2^{\prime}}$ & $R_{2}^{3^{\prime}}, R_{2}^{4^{\prime}}$ & $R_{2}^{3}, R_{2}^{4}$ & $R_{2}^{1}, R_{2}^{2}$ \\
\hline$R_{1}^{3}, R_{1}^{4}$ & $o_{1}$ & $o_{2}$ & $o_{1}$ & $o_{1}$ \\
\hline$R_{1}^{1}, R_{1}^{2}$ & $o_{2}$ & $o_{1}$ & $o_{1}$ & $o_{1}$ \\
\hline$R_{1}^{1^{\prime}}, R_{1}^{2^{\prime}}$ & $o_{2}$ & $o_{2}$ & $o_{1}$ & $o_{2}$ \\
\hline$R_{1}^{3^{\prime}}, R_{1}^{4^{\prime}}$ & $o_{2}$ & $o_{2}$ & $o_{2}$ & $o_{1}$ \\
\hline
\end{tabular}

Table 3. The social choice function $f$ defined on $\mathcal{S}^{2}$.

Note that in the direct revelation game induced by this social choice function, no agent has a dominant strategy. Hence, the rule is not strategy-proof (thus, violating immunity to both credible and (type 1) credible deviations). Also notice that the grand coalition has no profitable deviation. Hence, all profitable deviations involve a single agent, and for each one of them, the remaining agent can respond with a new profitable deviation. Hence, the social choice function is immune to (type 2) credible deviations, even if not strategy-proof.

Definition 14 Let $f$ be a social choice function on $\mathcal{U}^{n}$. Let $R_{N} \in \mathcal{U}^{n}$ and $C \subseteq N$. We say that $R_{C}^{\prime} \in \mathcal{U}^{c}$ a profitable deviation of $C$ against $R_{N}$ is strongly credible if $f\left(R_{C}^{\prime}, R_{N \mid C}\right) R_{i} f\left(\bar{R}_{S}, R_{C \backslash S}^{\prime}, R_{N \backslash C}\right)$ for all $S \subseteq C$, for all $\bar{R}_{S} \in \mathcal{U}^{s}$ and for some $i \in S$. A social choice function $f$ on $\mathcal{U}^{n}$ is immune to strongly credible deviations if for any $R_{N} \in \mathcal{U}^{n}$, any $C \subseteq N$, there is no strongly credible profitable deviation of $C$ against $R_{N}$.

\footnotetext{
${ }^{12}$ This example can be straightforwardly generalized when agents have differents sets of preferences: Let $\mathcal{R}_{1}=\left\{R^{1}, R^{2}, R^{3}, R^{4}\right\}, \mathcal{R}_{2}=\left\{R^{1^{\prime}}, R^{2^{\prime}}, R^{3^{\prime}}, R^{4 \prime}\right\}$ in Table 2 , and $f$ defined by the first two rows and columns in Table 3 .
} 
Proposition 8 All generalized Condorcet winner rules are immune to strongly credible deviations.

Let us say that we are aware that the idea of credibility may have other expressions. As already noted in the Introduction, Bernheim, Peleg, and Whinston (1986) introduced the concept of coalition-proof Nash equilibrium, and Peleg and Sudhölter (1999) studied its application to the set of strategy-proof voting rules in multidimensional single-peaked preference domains. This equilibrium notion turns out not to be discriminating, since all Generalized Median Voter Schemes satisfy it. That conclusion is the same as the one we obtain under our notion of strong credibility (Proposition 8) although they obtain it under weaker assumptions: we assume anonymity and ontoness. Peleg (1998) and Peleg and Sudhölter (1999) proposed the notion of strong coalition-proofness. The latter paper comments, by means of an example, that not all strategy-proof rules defined on the multidimensional single-peaked domain satisfy this condition. One can show that, in spite of its complicated formulation, their notion of strong coalition-proofness essentially boils down to requiring our basic notion of immunity to credible deviations in our context. ${ }^{13}$ Hence, we can read their example as an announcement that there is room for the analysis we have just provided, identifying and characterizing those functions among generalized Condorcet winner rules that satisfy these conditions and those that do not. Another related paper is due to Serizawa (2006), who defines an immunity notion in the line in our paper that only considers profitable deviations by pairs of agents.

Alternative definitions of credibility could also be obtained by weakening the notion of a profitable deviation allowing some, though not all, deviators to report their original preference. In this case, the new set of profitable deviations would be larger than ours. However, the resulting immunity notion would be equivalent to the one we use (see Definitions 5 or 14). Also notice that if indifferences are allowed one could also consider additional types of deviations and allowing some of the agents in the deviating coalition to weakly gain by deviating jointly. We do not go further in this direction because in our application all preferences are strict (see Serizawa, 2006).

\section{$5 \quad$ Final remarks}

We have studied the incentives of groups of agents to cooperate in manipulating social choice functions, by formalizing different notions of credibility, and characterized subclasses of strategy-proof rules that may be immune to credible manipulations by groups in multidimensional single-peaked preference domains.

The voting rules we have identified are interesting in several respects.

One interesting aspect is efficiency. It is clear that strategy-proof rules cannot be fully efficient unless they satisfy a strong notion of group strategy-proofness. Yet, those that satisfy our intermediate property have the interesting feature that any departure from their prescribed outcomes leading to an efficient one would not be credible. Thus, they are, in that sense, efficient up to credibility constraints.

\footnotetext{
${ }^{13}$ For a formal proof of this, see the proof of Proposition 9 in the Appendix.
} 
Another interesting conclusion of our analysis is that those rules that imply extreme distributions of voting power are immune to credible deviations from truth-telling. One could think that this distribution is uneven or unfair. However, the class of Generalized Condorcet winner rules that are obtained when the definitional parameters are concentrated in a single point do coincide, in each dimension, with those characterized by Thomson (1993, 1999) as being the only methods that satisfy an attractive normative property. His property, that Thomson calls "welfare domination under preference replacement", requires that when one agent changes preferences and modifies the social outcome, all other agents' welfare must change in the same direction. Hence, we not only found exactly what are the conditions that allow immunity, but also discovered that they may be partially justified in terms of preexisting normative concepts.

Finally, let us acknowledge that the treatment of strategic considerations by the different agents is somewhat asymmetric. Indeed, groups are allowed to form in order to manipulate, but our main concept of credibility only considers single-agent non-cooperative departures from cooperative agreements, à la Nash. This invites for further reflection regarding these and other issues of coalition formation, that we hope to keep developing in further work.

\section{Ackowledgements}

We thank the two referees, Matthew Jackson, and Albin Erlanson for their helpful suggestions. S. Barberà acknowledges financial support through grants ECO2014-53052$\mathrm{P}$ and SGR2014-515, and Severo Ochoa Programme for Centres of Excellence in R\&D (SEV-2015-0563). D. Berga acknowledges the support from grants ECO2013-45395-R and 2014-SGR-1360. B. Moreno acknowledges financial support from grants SEJ4941, SEJ-5980, and ECO2014-53767. D. Berga and B. Moreno thank the MOMA network under project ECO2014-57673-REDT.

\section{Appendix}

We first present the proofs of Claim 2, and Lemmas 2 and 3 used in the proofs of Proposition 4 and 5 in Section 3.2. For that, we need Definition 15.

Definition 15 Let $i \in N, R_{i} \in \mathcal{S}, k \in \mathcal{K}$, let $\mathcal{S}_{k}\left(R_{i}\right)=\left\{R_{i}^{k} \in \mathcal{S}_{B_{k}}: \tau\left(R_{i}^{k}\right)=\tau_{k}\left(R_{i}\right)\right\}$ where $\mathcal{S}_{B_{k}}$ is the set of all unidimensional (strict) single-peaked preferences on $B_{k}$ and $\tau\left(R_{i}^{k}\right)$ is the best alternative of $R_{i}^{k}$ in $B_{k}$.

We call a $R_{i}^{k} \in \mathcal{S}_{k}\left(R_{i}\right) \subseteq \mathcal{S}_{B_{k}}$ a unidimensional single-peaked preference on $B_{k}$ induced from $R_{i}$.

Proof of Claim 2 Let $f$ be a generalized Condorcet winner rule and let $R_{C}^{\prime}$ be a profitable deviation of $C$ against $R_{N}$ for $f$. To prove part (i), define $\overline{\mathcal{K}}=\left\{k \in \mathcal{K}: f_{k}\left(R_{C}^{\prime}, R_{N \mid C}\right) \neq f_{k}\left(R_{N}\right)\right\}$. Without loss of generality, let $\overline{\mathcal{K}}=\{1, \ldots, \bar{k}\}, \bar{k}$ denoting its cardinality. By contradiction, suppose that there exists $i \in C$ such that for any $k \in \overline{\mathcal{K}}$, agent $i$ is not winning according 
to $R_{i}$ at $\left(R_{C}^{\prime}, R_{N \backslash C}\right)$ in dimension $k$. That is, agent $i$ is losing at $\left(R_{C}^{\prime}, R_{N \backslash C}\right)$ in dimension $k$ : $\left(f_{k}\left(R_{N}\right), f_{\mathcal{K} \backslash\{k\}}\left(R_{C}^{\prime}, R_{N \backslash C}\right)\right) P_{i} f\left(R_{C}^{\prime}, R_{N \backslash C}\right)$. Let $\widehat{R}_{i}$ be defined by Claim 1 given $x=$ $f\left(R_{C}^{\prime}, R_{N \backslash C}\right)$. By part (2) of Claim 1, we have that for any $k \in \overline{\mathcal{K}},\left(f_{k}\left(R_{N}\right), f_{\mathcal{K} \backslash\{k\}}\left(R_{C}^{\prime}, R_{N \backslash C}\right)\right)$ $\widehat{P}_{i} f\left(R_{C}^{\prime}, R_{N \backslash C}\right)$. In particular, for $k=1$, we obtain $\left(f_{1}\left(R_{N}\right), f_{\mathcal{K} \backslash\{1\}}\left(R_{C}^{\prime}, R_{N \backslash C}\right)\right) \widehat{P}_{i} f\left(R_{C}^{\prime}, R_{N \backslash C}\right)$. Now, proceed as follows: define $\bar{k}-1$ steps and in each step $t \in\{1, \ldots, \bar{k}-1\}$ replace $f_{t+1}\left(R_{C}^{\prime}, R_{N \backslash C}\right)$ by $f_{t+1}\left(R_{N}\right)$ in $\left(f_{t}\left(R_{N}\right), f_{\{1, \ldots, t-1\}}\left(R_{N}\right), f_{\overline{\mathcal{K}} \backslash\{t+1, \ldots, \bar{k}\}}\left(R_{C}^{\prime}, R_{N \backslash C}\right), f_{\mathcal{K} \backslash \overline{\mathcal{K}}}\left(R_{C}^{\prime}, R_{N \backslash C}\right)\right)$. By transitivity of preferences we will obtain that $f\left(R_{N}\right) \widehat{P}_{i} f\left(R_{C}^{\prime}, R_{N \backslash C}\right)$ which will be the desired contradiction. To check the contradiction, note that by part (1) in Claim 1 and by tops-onliness of the generalized Condorcet winner rule $f$, if $R_{C}^{\prime}$ is a profitable deviation of $C$ against $R_{N}, R_{C}^{\prime}$ is also a profitable deviation of $C$ against $\left(\widehat{R}_{C}, R_{N \backslash C}\right)$.

Consider the first step, $t=1$, and change $f_{2}\left(R_{C}^{\prime}, R_{N \backslash C}\right)$ by $f_{2}\left(R_{N}\right)$. By part (3) of Claim 1 applied for $k=2, z_{\mathcal{K} \backslash\{2\}}=\left(f_{1}\left(R_{N}\right), f_{\mathcal{K} \backslash\{1,2\}}\left(R_{C}^{\prime}, R_{N \backslash C}\right)\right)$ and $w_{\mathcal{K} \backslash\{2\}}=f_{\mathcal{K} \backslash\{2\}}\left(R_{C}^{\prime}, R_{N \backslash C}\right)$ we obtain the following:

$$
\left(f_{2}\left(R_{N}\right), z_{\mathcal{K} \backslash\{2\}}\right) \widehat{P}_{i}\left(f_{2}\left(R_{C}^{\prime}, R_{N \backslash C}\right), z_{\mathcal{K} \backslash\{2\}}\right) \Leftrightarrow\left(f_{2}\left(R_{N}\right), w_{\mathcal{K} \backslash\{2\}}\right) \widehat{P}_{i}\left(f_{2}\left(R_{C}^{\prime}, R_{N \backslash C}\right), w_{\mathcal{K} \backslash\{2\}}\right) .
$$

Note that $\left(f_{2}\left(R_{N}\right), w_{\mathcal{K} \backslash\{2\}}\right) \widehat{P}_{i}\left(f_{2}\left(R_{C}^{\prime}, R_{N \backslash C}\right), w_{\mathcal{K} \backslash\{2\}}\right)$ (equivalently, $\left(f_{2}\left(R_{N}\right), f_{\mathcal{K} \backslash\{2\}}\left(R_{C}^{\prime}, R_{N \backslash C}\right)\right.$ ) $\left.\widehat{P}_{i} f\left(R_{C}^{\prime}, R_{N \backslash C}\right)\right)$ holds since by part (2) of Claim 1 applied for $k=2, z_{2}=f_{2}\left(R_{N}\right)$, $w_{2}=f_{2}\left(R_{C}^{\prime}, R_{N \backslash C}\right)$ we obtain the following:

$$
\left(f_{2}\left(R_{N}\right), f_{\mathcal{K} \backslash\{2\}}\left(R_{C}^{\prime}, R_{N \backslash C}\right)\right) \widehat{P}_{i} f\left(R_{C}^{\prime}, R_{N \backslash C}\right) \Leftrightarrow\left(f_{2}\left(R_{N}\right), f_{\mathcal{K} \backslash\{2\}}\left(R_{C}^{\prime}, R_{N \backslash C}\right)\right) P_{i} f\left(R_{C}^{\prime}, R_{N \backslash C}\right) .
$$

And moreover, the latter strictness preference relationship holds by hypothesis at the beginning of this proof. Thus, we get that

$$
\left(f_{2}\left(R_{N}\right), f_{1}\left(R_{N}\right), f_{\mathcal{K} \backslash\{1,2\}}\left(R_{C}^{\prime}, R_{N \backslash C}\right)\right) \widehat{P}_{i}\left(f_{2}\left(R_{C}^{\prime}, R_{N \backslash C}\right), f_{1}\left(R_{N}\right), f_{\mathcal{K} \backslash\{1,2\}}\left(R_{C}^{\prime}, R_{N \backslash C}\right)\right) .
$$

Repeating exactly the same argument for any $t \in\{2, \ldots, \bar{k}-1\}$, we will obtain our desired contradiction: $f\left(R_{N}\right) \widehat{P}_{i} f\left(R_{C}^{\prime}, R_{N \backslash C}\right)$ which ends the proof of part (i).

To prove part (ii), by contradiction let $i \in C$ be winning at $\left(R_{C}^{\prime}, R_{N \backslash C}\right)$ in some dimension $k$ and suppose that for any other agent $j \in C \backslash\{i\}, j$ is also winning at $\left(R_{C}^{\prime}, R_{N \backslash C}\right)$ in $k$. That is, suppose that for any $j \in C \backslash\{i\}, f\left(R_{C}^{\prime}, R_{N \backslash C}\right) P_{j}\left(f_{k}\left(R_{N}\right), f_{\mathcal{K} \backslash\{k\}}\left(R_{C}^{\prime}, R_{N \backslash C}\right)\right)$ holds.

We now proceed to define unidimensional single-peaked preferences for dimension $k$ using Definition 15 as follows: for any $j \in N \backslash C$, let $R_{j}^{k} \in \mathcal{S}_{k}\left(R_{j}\right)$; for any $i \in C$, let $R_{i}^{\prime k} \in \mathcal{S}_{k}\left(R_{i}^{\prime}\right)$, and for any $l \in C$ let $R_{l}^{k} \in \mathcal{S}_{k}\left(R_{l}\right)$ such that for any $x_{k}, y_{k} \in B_{k}$,

$$
x_{k} P_{l}^{k} y_{k} \Leftrightarrow\left(x_{k}, f_{\mathcal{K} \backslash\{k\}}\left(R_{C}^{\prime}, R_{N \backslash C}\right)\right) P_{l}\left(y_{k}, f_{\mathcal{K} \backslash\{k\}}\left(R_{C}^{\prime}, R_{N \backslash C}\right)\right) .
$$

Note that $R_{l}^{k}$ is well-defined. Let $x_{k}=f_{k}\left(R_{C}^{\prime}, R_{N \backslash C}\right)$ and $y_{k}=f_{k}\left(R_{N}\right)$. Then, we obtain by definition of $R_{l}^{k}$ that for any $j \in C \backslash\{i\}, f_{k}\left(R_{C}^{\prime}, R_{N \backslash C}\right) P_{l}^{k} f_{k}\left(R_{N}\right)$. By Definition 10, by any $l \in C, F_{k}\left(R_{C}^{\prime k}, R_{N \backslash C}^{k}\right) P_{l}^{k} F_{k}\left(R_{N}^{k}\right)$. Observe that this last expression is a contradiction to group strategy-proofness of generalized Condorcet winner rules. This ends the proof of part (ii). 
To prove part (iii), by contradiction suppose that there exists $k \in \mathcal{K}$ such that for any agent $i \in C, f\left(R_{C}^{\prime}, R_{N \backslash C}\right) P_{i}\left(f_{k}\left(R_{N}\right), f_{\mathcal{K} \backslash\{k\}}\left(R_{C}^{\prime}, R_{N \backslash C}\right)\right)\left(^{*}\right)$. Now, by Definition 15 , let $R_{i}^{k}, R_{i}^{\prime k}$ be the induced one dimensional preferences on $B_{k}$ as follows:

For $i \in N \backslash C$, take any $R_{i}^{k} \in \mathcal{S}_{k}\left(R_{i}\right)$. For $i \in C$, take $R_{i}^{\prime k} \in \mathcal{S}_{k}\left(R_{i}^{\prime}\right)$. For $i \in C$, take $R_{i}^{k} \in \mathcal{S}_{k}\left(R_{i}\right)$ such that $x=f\left(R_{C}^{\prime}, R_{N \backslash C}\right)$. That is, for any $x_{k}, y_{k} \in B_{k}$,

$$
y_{k} P_{i}^{k} x_{k} \Leftrightarrow\left(y_{k}, f_{\mathcal{K} \backslash\{k\}}\left(R_{C}^{\prime}, R_{N \backslash C}\right)\right) P_{i}^{k}\left(x_{k}, f_{\mathcal{K} \backslash\{k\}}\left(R_{C}^{\prime}, R_{N \backslash C}\right)\right) .
$$

Observe that by Definition $10, F_{k}\left(R_{N}^{k}\right)=f_{k}\left(R_{N}\right)$ and $F_{k}\left(R_{C}^{\prime k}, R_{N \backslash C}^{k}\right)=f_{k}\left(R_{C}^{\prime}, R_{N \backslash C}\right)$. Thus, combining the latter equality with expression $\left(^{*}\right)$, we obtain that for any $k$ and any agent $i \in C, F_{k}\left(R_{C}^{\prime}, R_{N \backslash C}\right) P_{i}^{k} F_{k}\left(R_{N}\right)$ holds. And this contradicts that $F_{k}$ be a group strategyproof. This end the proof of part (iii).

Proof of Lemma 2 Let $f$ be a generalized Condorcet winner rule and let $R_{C}^{\prime}$ be a profitable deviation of $C$ against $R_{N}$ for $f$. Consider, for each dimension $k$, the sets $L_{k} \equiv$ $L_{k}\left(f, R_{C^{\prime}}, R_{N}\right)$ and $W_{k} \equiv W_{k}\left(f, R_{C^{\prime}}, R_{N}\right)$, that is, the partition of agents in $C$ defined by the ones winning and the ones losing at $\left(R_{C}^{\prime}, R_{N \backslash C}\right)$ in dimension $k$ (see Definition 11). By part (i) of Claim 2 for any agent in $C$ there is $k \in \mathcal{K}$ such that $i \in W_{k}$. By part (ii) of Claim 2 there exists a different agent $j$ in $C$ such that $j \in L_{k}$. Again, by part (i) of Claim $2, j \in W_{k^{\prime}}$ for some $k^{\prime} \in \mathcal{K} \backslash\{k\}$. If some agent $i \in W_{k}$ belongs to $L_{k^{\prime}}$, the result holds.

Otherwise, suppose that for any $i \in W_{k}, i \notin L_{k^{\prime}}$. Since $L_{k} \cup W_{k}=L_{k^{\prime}} \cup W_{k^{\prime}}=C$, then $W_{k} \varsubsetneqq W_{k^{\prime}}$ and $L_{k^{\prime}} \varsubsetneqq L_{k}$ (note that $L_{k^{\prime}} \neq L_{k}$ since $j \in L_{k} \cap W_{k^{\prime}}$ ). Take now an agent $l \in L_{k^{\prime}}$ which exists by part (ii) of Claim 2. By part (i) of Claim $2, l \in W_{k^{\prime \prime}}$ for some $k^{\prime \prime} \in \mathcal{K}$. If some agents $i \in W_{k^{\prime}}, i \in L_{k^{\prime \prime}}$, then the result holds.

Otherwise, suppose that for any $i \in W_{k^{\prime}}, i \notin L_{k^{\prime \prime}}$. Since $L_{k^{\prime}} \cup W_{k^{\prime}}=L_{k^{\prime \prime}} \cup W_{k^{\prime \prime}}=C$, then $W_{k^{\prime}} \varsubsetneqq W_{k^{\prime \prime}}$ and $L_{k^{\prime \prime}} \varsubsetneqq L_{k^{\prime}} \varsubsetneqq L_{k}$ (note that $L_{k^{\prime \prime}} \neq L_{k^{\prime}}$ since $j \in L_{k^{\prime}} \cap W_{k^{\prime \prime}}$ ). Since there is a finite number of agents in $L_{k}$ and a finite number of $k$, we will obtain the result for some $k$. Otherwise, there would be a $k^{*}$ such that $L_{k *}=\varnothing$ which is a contradiction to part (iii) of Claim 2.

Proof of Lemma 3 Let $f$ be a generalized Condorcet winner rule, $R_{C}^{\prime}$ be a profitable deviation of $C$ against $R_{N}$ for $f$ and $k$ be such that $P_{k}(f)$ is degenerate. Since $i \in W_{k}$, then $f_{k}\left(R_{C}^{\prime}, R_{N \backslash C}\right) \neq f_{k}\left(R_{N}\right)$. Consider two cases.

In the first case, in $R_{N}$ each agent' $k$-dimensional top is placed in the same side of the parameters' unique position. Without loss of generality, assume that the $k$-dimensional tops are to the left of the parameter. Note that $f_{k}\left(R_{N}\right)$ is the top closest to the single parameter. Since $i \in W_{k}$, then $f_{k}\left(R_{C}^{\prime}, R_{N \backslash C}\right)<f_{k}\left(R_{N}\right)$. Otherwise, if $f_{k}\left(R_{C}^{\prime}, R_{N \backslash C}\right)>f_{k}\left(R_{N}\right)$, by singlepeakedness ${ }^{14}$, we obtain $\left(f_{k}\left(R_{N}\right), f_{\mathcal{K} \backslash\{k\}}\left(R_{C}^{\prime}, R_{N \backslash C}\right)\right) P_{i}\left(f_{k}\left(R_{C}^{\prime}, R_{N \backslash C}\right), f_{\mathcal{K} \backslash\{k\}}\left(R_{C}^{\prime}, R_{N \backslash C}\right)\right)$ contradicting that $i \in W_{k}$. Then, by definition of $f$, for any $l \in N$ such that $\tau_{k}\left(R_{l}\right)=f_{k}\left(R_{N}\right)$ then $l \in C$ and $\tau_{k}\left(R_{l}^{\prime}\right) \leq f_{k}\left(R_{C}^{\prime}, R_{N \backslash C}\right)<f_{k}\left(R_{N}\right)$. And, by single-peakedness, for any such $l \in C,\left(f_{k}\left(R_{N}\right), f_{\mathcal{K} \backslash\{k\}}\left(R_{C}^{\prime}, R_{N \backslash C}\right)\right) P_{l}\left(f_{k}\left(R_{C}^{\prime}, R_{N \backslash C}\right), f_{\mathcal{K} \backslash\{k\}}\left(R_{C}^{\prime}, R_{N \backslash C}\right)\right)$. Take any of such agents, say $j \in C$. Take profile $\left(R_{C}^{\prime}, R_{N \backslash C}\right)$ and let $j \in C$ announce $\bar{R}_{j}$ such

\footnotetext{
${ }^{14}$ In this proof and that of Proposition 8, when we say "by single-peakedness" we mean both, unidimensional single-peaked on $B_{k}$ and multidimensional single-peaked on $B$.
} 
that $\tau_{k}\left(\bar{R}_{j}\right)=\tau_{k}\left(R_{j}\right)$ and for each $k^{\prime} \in \mathcal{K} \backslash\{k\}, \tau_{k^{\prime}}\left(\bar{R}_{j}\right)=\tau_{k^{\prime}}\left(R_{j}^{\prime}\right)$. By definition of $f$, $f_{k}\left(\bar{R}_{j}, R_{C \backslash\{j\}}^{\prime}, R_{N \backslash C}\right)=f_{k}\left(R_{N}\right)$ and $f_{\mathcal{K} \backslash\{k\}}\left(\bar{R}_{j}, R_{C \backslash\{j\}}^{\prime}, R_{N \backslash C}\right)=f_{\mathcal{K} \backslash\{k\}}\left(R_{C}^{\prime}, R_{N \backslash C}\right)$. Therefore, $f\left(\bar{R}_{j}, R_{C \backslash\{j\}}^{\prime}, R_{N \backslash C}\right) P_{j} f\left(R_{C}^{\prime}, R_{N \backslash C}\right)$ by single-peakedness, which means that $R_{C}^{\prime}$ is not a credible profitable deviation.

In the second case, in both sides of the single parameter there is at least one agents' top given $R_{N}$. Thus, $f_{k}\left(R_{N}\right)$ is the single parameter. Suppose, without loss of generality, that $f_{k}\left(R_{C}^{\prime}, R_{N \backslash C}\right)<f_{k}\left(R_{N}\right)$. Observe that by definition of $f$, there exists $j \in C$ such that $\tau_{k}\left(R_{j}^{\prime}\right)<f_{k}\left(R_{C}^{\prime}, R_{N \backslash C}\right)<\tau_{k}\left(R_{j}\right)$. Then, by single-peakedness, for agent $j \in C$, $\left(f_{k}\left(R_{N}\right), f_{\mathcal{K} \backslash\{k\}}\left(R_{C}^{\prime}, R_{N \backslash C}\right)\right) P_{j}\left(f_{k}\left(R_{C}^{\prime}, R_{N \backslash C}\right), f_{\mathcal{K} \backslash\{k\}}\left(R_{C}^{\prime}, R_{N \backslash C}\right)\right)$. As in the above case, take profile $\left(R_{C}^{\prime}, R_{N \backslash C}\right)$ and let agent $j \in C$ announce $\bar{R}_{j}$ such that $\tau_{k}\left(\bar{R}_{j}\right)=\tau_{k}\left(R_{j}\right)$ and for each $k^{\prime} \in \mathcal{K} \backslash\{k\}, \tau_{k^{\prime}}\left(\bar{R}_{j}\right)=\tau_{k^{\prime}}\left(R_{j}^{\prime}\right)$. By definition of $f, f_{k}\left(\bar{R}_{j}, R_{C \backslash\{j\}}^{\prime}, R_{N \backslash C}\right)=f_{k}\left(R_{N}\right)$ and $f_{\mathcal{K} \backslash\{k\}}\left(\bar{R}_{j}, R_{C \backslash\{j\}}^{\prime}, R_{N \backslash C}\right)=f_{\mathcal{K} \backslash\{k\}}\left(R_{C}^{\prime}, R_{N \backslash C}\right)$. Thus, $f\left(\bar{R}_{j}, R_{C \backslash\{j\}}^{\prime}, R_{N \backslash C}\right) P_{j} f\left(R_{C}^{\prime}, R_{N \backslash C}\right)$ by single-peakedness meaning that $R_{C}^{\prime}$ is not a credible profitable deviation. This ends the proof.

We now prove Propositions 6, 7, and 8 stated in Section 4.

Proof of Proposition 6 By definition immunity to (type 1) credible deviations implies immunity to credible deviations. To prove the converse, let $R_{N} \in \mathcal{U}^{n}, C \subseteq N$, and $R_{C}^{\prime} \in \mathcal{U}^{c}$ be a profitable deviation of $C$ against $R_{N}$. Suppose that for all $i \in C$, $f\left(R_{C}^{\prime}, R_{N \mid C}\right) R_{i} f\left(R_{i}, R_{C \backslash\{i\}}^{\prime}, R_{N \backslash C}\right)$. By Lemma $1 f$ is strategy-proof, thus $f\left(R_{C}^{\prime}, R_{N \mid C}\right) I_{i}$ $f\left(R_{i}, R_{C \backslash\{i\}}^{\prime}, R_{N \backslash C}\right)$ for all $i \in C$. By immunity to credible deviations, there exists $i \in C$ such that $f\left(\bar{R}_{i}, R_{C \backslash\{i\}}^{\prime}, R_{N \backslash C}\right) P_{i} f\left(R_{C}^{\prime}, R_{N \mid C}\right)$ for some $\bar{R}_{i} \in \mathcal{U}$. By these two facts, for some $i \in C, f\left(\bar{R}_{i}, R_{C \backslash\{i\}}^{\prime}, R_{N \backslash C}\right) P_{i} f\left(R_{i}, R_{C \backslash\{i\}}^{\prime}, R_{N \backslash C}\right)$ which contradicts strategy-proofness.

Proof of Proposition $7 \quad$ By definition immunity to credible deviations implies immunity to (type 2) credible deviations. To prove the converse, let $R_{N} \in \mathcal{U}^{n}, C \subseteq N$, and $R_{C}^{\prime} \in \mathcal{U}^{c}$ be a profitable deviation of $C$ against $R_{N}$. Suppose that for all $i \in C$, $f\left(R_{C}^{\prime}, R_{N \backslash C}\right) R_{i} f\left(\bar{R}_{i}, R_{C \backslash\{i\}}^{\prime}, R_{N \backslash C}\right)$ for all $\bar{R}_{i} \in \mathcal{U}$. Thus, $f\left(R_{C}^{\prime}, R_{N \backslash C}\right) R_{i} f\left(R_{i}, R_{C \backslash\{i\}}^{\prime}, R_{N \backslash C}\right)$ for all $i \in C$. By immunity to (type 2) credible deviations, there exists $i \in N$ such that $f\left(\bar{R}_{i}, R_{C \backslash\{i\}}^{\prime}, R_{N \backslash(C \cup\{i\})}\right) P_{i} f\left(R_{C}^{\prime}, R_{N \backslash C}\right)$ for some $\bar{R}_{i} \in \mathcal{U}$. Suppose that agent $i \in C$. Since $f$ is strategy-proof, $f\left(R_{C}^{\prime}, R_{N \backslash C}\right) I_{i} f\left(R_{i}, R_{C \backslash\{i\}}^{\prime}, R_{N \backslash C}\right)$ for all $i \in C$. Then, we have that for some $i \in C, f\left(\bar{R}_{i}, R_{C \backslash\{i\}}^{\prime}, R_{N \backslash C}\right) P_{i} f\left(R_{i}, R_{C \backslash\{i\}}^{\prime}, R_{N \backslash C}\right)$ which contradicts strategy-proofness. Therefore, it must be that agent $i \in N \backslash C$. Thus, $f\left(R_{i}, R_{C}^{\prime}, R_{N \backslash(C \cup\{i\})}\right) R_{i} f\left(\bar{R}_{i}, R_{C}^{\prime}, R_{N \backslash(C \cup\{i\})}\right)$ by strategy-proofness, and therefore we obtain $f\left(R_{i}, R_{C}^{\prime}, R_{N \backslash(C \cup\{i\})}\right) P_{i} f\left(R_{C}^{\prime}, R_{N \backslash C}\right)$ for some $i \in N \backslash C$ which contradicts that $f$ is single-valued and $R_{i}^{\prime}=R_{i}$.

Proof of Proposition 8 Let $f$ be a generalized Condorcet winner rule with lists of parameters, denoted in each dimension $k$ as $p_{k}^{-} \leq p_{k}^{+}$. To prove that $f$ is immune to strongly credible deviations, let $R_{N} \in \mathcal{S}^{n}, C \subseteq N$, and $R_{C}^{\prime} \in \mathcal{S}^{c}$ be a profitable deviation of $C$ against $R_{N}$. Since $R_{C}^{\prime}$ is a profitable deviation, by part (i) of Claim 2, there must exist at least one dimension $k$ in which $f_{k}\left(R_{N}\right) \neq f_{k}\left(R_{C}^{\prime}, R_{N \backslash C}\right)$ and some agent $i \in C$ is winning. By part (ii) of Claim 2, there is an agent $j \in C \backslash\{i\}$ who is losing in that dimension $k$. Let $\bar{C}$ and $\widetilde{C}$ be a 
partition of $C$ such that $\bar{C}=\{i \in C$ : is winning in dimension $k\}$ and $\widetilde{C}=\{j \in C$ : is losing in dimension $k\}$. Suppose, without loss of generality, that $f_{k}\left(R_{C}^{\prime}, R_{N \backslash C}\right)<f_{k}\left(R_{N}\right)$. By definition of $\bar{C}$, for any $i \in \bar{C}, \tau_{k}\left(R_{i}\right)<f_{k}\left(R_{N}\right)$. By definition of $\widetilde{C}, \tau_{k}\left(R_{j}\right)>f_{k}\left(R_{C}^{\prime}, R_{N \backslash C}\right)$. We distinguish two cases:

Case 1. For any $j \in \widetilde{C}, \tau_{k}\left(R_{j}\right) \leq f_{k}\left(R_{N}\right)$. Since $f_{k}\left(R_{C}^{\prime}, R_{N \backslash C}\right)<f_{k}\left(R_{N}\right)$, then for any $l \in C, \tau_{k}\left(R_{l}^{\prime}\right) \leq f_{k}\left(R_{N}\right)$. Also, it must happen that for some $j \in \widetilde{C}, \tau_{k}\left(R_{j}\right)=f_{k}\left(R_{N}\right)$. Let $S=\left\{j \in \widetilde{C}: \tau_{k}\left(R_{j}\right)=f_{k}\left(R_{N}\right)\right\}$. Then for any $l \in S$, let $\bar{R}_{l}$ be such that $\tau_{k}\left(\bar{R}_{l}\right)=\tau_{k}\left(R_{l}\right)$ and $\tau_{k^{\prime}}\left(\bar{R}_{l}\right)=\tau_{k^{\prime}}\left(R_{l}^{\prime}\right)$ for any $k^{\prime} \in \mathcal{K} \backslash\{k\}$. Thus $f_{k}\left(\bar{R}_{S}, R_{C \backslash S}^{\prime}, R_{N \backslash C}\right)=f_{k}\left(R_{N}\right)$ and by single-peakedness $f\left(\bar{R}_{S}, R_{C \backslash S}^{\prime}, R_{N \backslash C}\right) P_{l} f\left(R_{C}^{\prime}, R_{N \backslash C}\right)$ for any $l \in S$. Which means that $R_{C}^{\prime}$ is not strongly credible.

Case 2. For some $j \in \widetilde{C}, \tau_{k}\left(R_{j}\right)>f_{k}\left(R_{N}\right)$. Let $S=\left\{j \in \widetilde{C}: \tau\left(R_{j}\right) \geq f_{k}\left(R_{N}\right)\right.$ and $\left.\tau_{k}\left(R_{l}^{\prime}\right) \neq \tau_{k}\left(R_{l}\right)\right\}$. Since $f_{k}\left(R_{C}^{\prime}, R_{N \backslash C}\right)<f_{k}\left(R_{N}\right), S$ is not empty. Then for any $l \in$ $S$, let $\bar{R}_{l}$ be such that $\tau_{k}\left(\bar{R}_{l}\right)=\tau_{k}\left(R_{l}\right)$ and $\tau_{k^{\prime}}\left(\bar{R}_{l}\right)=\tau_{k^{\prime}}\left(R_{l}^{\prime}\right)$ for any $k^{\prime} \in \mathcal{K} \backslash\{k\}$. By definition of $f, f_{k}\left(\bar{R}_{S}, R_{C \backslash S}^{\prime}, R_{N \backslash C}\right)=f_{k}\left(R_{N}\right)$ and for any $k^{\prime} \in \mathcal{K} \backslash\{k\}, f_{k^{\prime}}\left(\bar{R}_{S}, R_{C \backslash S}^{\prime}, R_{N \backslash C}\right)=$ $f_{k^{\prime}}\left(R_{C}^{\prime}, R_{N \backslash C}\right)$. Thus, by single-peakedness, $f\left(\bar{R}_{S}, R_{C \backslash S}^{\prime}, R_{N \backslash C}\right) P_{l} f\left(R_{C}^{\prime}, R_{N \backslash C}\right)$ for any $l \in S$, meaning that $R_{C}^{\prime}$ is not strongly credible.

As promised in Section 4, we now prove the equivalence in our context between strong coalition-proofness as defined by Peleg and Sudhölter (1999) and our immunity to credible deviations of $f$. To do so, we first introduce the notion of strong coalition-proofness for revelation games.

A revelation game in strategic form is a system $G\left(f, \widehat{R}_{N}\right)=\left(N, A,(\mathcal{U})_{i \in N}, f, \widehat{R}_{N}\right)$ where $N$ is the set of players, $A$ is the set of outcomes, $\mathcal{U}$ the (non-empty) set of strategies of each agent (the set of all possible preferences as defined in Section 2), $f$ is a function from preference profiles to $A$, and $\widehat{R}_{N}$ is a specific profile of preferences. Then, given a game $G\left(f, \widehat{R}_{N}\right)$, a coalition $C \subseteq N, C \neq \varnothing$, and a profile $\widetilde{R}_{N} \in \mathcal{U}^{n}$, the reduced game of $G\left(f, \widehat{R}_{N}\right)$ with respect

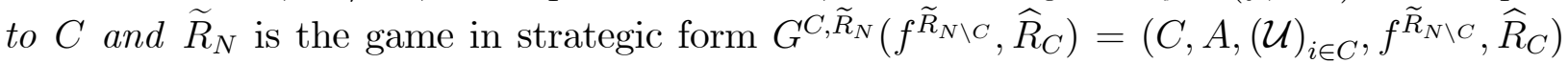
where $C$ is the set of players, $A$ is the set of outcomes, $\mathcal{U}$ the (non-empty) set of strategies of each agent, $f^{\widetilde{R}_{N \backslash C}}: \mathcal{U}^{c} \rightarrow A$ is a function such that $f^{\widetilde{R}_{N \backslash C}}\left(R_{C}\right)=f\left(R_{C}, \widetilde{R}_{N \backslash C}\right)$ for all $R_{C} \in \mathcal{U}^{c}$, and $\widehat{R}_{C}$ is the profile of preferences.

Definition 16 Let $G\left(f, \widehat{R}_{N}\right)=\left(N,(\mathcal{U})_{i \in N}, f, \widehat{R}_{N}\right)$ be a revelation strategic game. We say that $\widetilde{R}_{N} \in \mathcal{U}^{n}$ is a strong coalition-proof Nash equilibrium if (1) $\widetilde{R}_{N}$ is a Nash equilibrium of $G\left(f, \widehat{R}_{N}\right)$; and (2) for every $C \subseteq N, C \neq \varnothing$, and every Nash equilibrium $R_{C}^{\prime}$ of $G^{C, \widetilde{R}_{N}}\left(f^{\widetilde{R}_{N \backslash C}}, \widehat{R}_{C}\right)$, there exists $i \in C$ such that $f^{\widetilde{R}_{N \backslash C}}\left(\widetilde{R}_{C}\right)=f\left(\widetilde{R}_{N}\right) \widehat{R}_{i} f\left(R_{C}^{\prime}, \widetilde{R}_{N \backslash C}\right)=$ $f^{\widetilde{R}_{N \backslash C}}\left(R_{C}^{\prime}\right)$.

Definition 17 A social choice function $f$ on $\mathcal{U}^{n}$ is strong coalition-proof if for any $R_{N} \in$ $\mathcal{U}^{n}$ truthtelling is a strong coalition-proof Nash equilibrium of $G\left(f, R_{N}\right)$.

The strategy space for each agent is $\mathcal{U}$, and the outcome function is $f$. 
Proposition 9 Any strong coalition-proof social choice function $f$ is immune to credible deviations. The converse holds when best deviations exist.

Proof of Proposition 9 By contradiction, suppose that $f$ is not immune to credible deviations. That is, there exist $R_{N}$, and a deviation $R_{C}^{\prime}$ of $C$ against $R_{N}$ that is profitable, $f\left(R_{C}^{\prime}, R_{N \backslash C}\right) P_{i} f\left(R_{N}\right)$, and credible, $f\left(R_{C}^{\prime}, R_{N \backslash C}\right) R_{i} f\left(\bar{R}_{i}, R_{C \backslash\{i\}}^{\prime}, R_{N \backslash C}\right)$ for all $\bar{R}_{i} \in \mathcal{U}$ and for all $i \in C$. The latter implies that $R_{C}^{\prime}$ is a Nash equilibrium of the reduced game $G^{C, R_{N}}$. Since $f$ is strong coalition-proof, then $R_{N}$ is a strong coalition-proof Nash equilibrium of $G\left(f, R_{N}\right)$. By condition (2) in Definition 16 there must exist an agent $i \in C$ such that $f\left(R_{N}\right) R_{i} f\left(R_{C}^{\prime}, R_{N \backslash C}\right)$ contradicting that $R_{C}^{\prime}$ is a profitable deviation of $C$ against $R_{N}$.

To show the converse, assume that there exist best deviations. Let $f$ be immune to credible deviations and take any $R_{N}$. We have to show that truthtelling is a strong coalition-proof Nash equilibrium of $G\left(f, R_{N}\right)$. By Lemma $1, f$ is strategy-proof. Therefore, $R_{N}$ is a Nash equilibrium of $G\left(f, R_{N}\right)$ (part (1) in Definition 16). Take $C \subseteq N, C \neq \varnothing$, and any Nash equilibrium $R_{C}^{\prime}$ of $G^{C, R_{N}}$. Since $f$ is immune to credible deviations, no profitable deviation can be credible, that is, there exists an agent $i \in C$ such that $f\left(R_{N}\right) R_{i} f\left(R_{C}^{\prime}, R_{N \backslash C}\right)$. 


\section{References}

Barberà, S., Berga, D., Moreno B., 2010. Individual versus group strategy-proofness: when do they coincide?. Journal of Economic Theory 145, 1648-1674.

Barberà, S., Berga, D., Moreno, B., 2016. Group strategy-proofness in private good economies. American Economic Review 106, 1073-1099.

Barberà, S., Gul, F., Stacchetti, E., 1993. Generalized Median Voter Schemes and Committees. J Econ Theory 61, 262-289.

Barberà, S., Sonnenschein, H., Zhou, L., 1991. Voting by committees. Econometrica 59, 595-609.

Bernheim, B. D., Peleg, B., Whinston, M. D., 1987. Coalition-proof Nash equilibria. I. Concepts. Journal of Economic Theory 42, 1-12.

Border, K., Jordan, J., 1983. Straightforward elections, unanimity and phantom voters. Rev. Econ. Stud. 50, 153-70.

Le Breton, M., Sen, A., 1999. Separable preferences, decomposability and strategyproofness. Econometrica 67, 605-28.

Le Breton, M., Weymark, J., 1999. Strategy-proof social choice with continuous separable preferences. Journal of Mathematical Economics 32, 47-85.

Le Breton, M., Zaporozhets, V., 2009. On the Equivalence of Coalitional and Individual Strategy-Proofness Properties. Soc. Choice Welfare 33, 287-309.

Moulin, H., 1980. On Strategy-proofness and Single Peakedness. Pub Choice 35, 437-455. Peleg, B., 1998. Almost all equilibiria in dominant strategies are coalition-proof. Econom. Lett. 60, 157-162.

Peleg, B., Sudhölter, P., 1999. Single-peakedness and coalition-proofness. Rev. Econ. Design 4, 381-387.

Serizawa, S., 2006. Pairwise strategy-proofness and self-enforcing manipulation. Soc Choice Welfare 26, 305-331.

Thomson, W., 1993. The replacement principle in public good economies with single-peaked preferences. Economics Letters 42, 31-36.

Thomson, W., 1999. Welfare-domination under preference-replacement: A survey and open questions. Soc Choice Welfare 16, 373-394. 